\title{
Bethe Ansatz for the Universal Weight Function
}

\author{
Luc Frappat, Sergey Khoroshkin, Stanislav Pakuliak and \\ Éric Ragoucy
}

\begin{abstract}
We consider universal off-shell Bethe vectors given in terms of Drinfeld realization of the algebra $\bar{U}_{q}\left(\widehat{\mathfrak{g l}}_{N}\right)[10,12]$. We investigate ordering properties of the product of the transfer matrix and these vectors. We derive that these vectors are eigenvectors of the transfer matrix if their Bethe parameters satisfy the universal Bethe equations [1].
\end{abstract}

\section{Introduction}

Algebraic Bethe ansatz for quantum integrable models with $\mathfrak{g l}_{2}$ symmetry as well as hierarchical Bethe ansatz for models with higher rank symmetries solves the eigenvalue problem for the set of the commuting quantum integrals of motion. The eigenvectors in these methods are built from the matrix elements of the monodromy matrix which satisfies Yang-Baxter relation defined by the quantum Rmatrix. Quantum integrable models solvable by these methods correspond to the different monodromy matrices and quantum R-matrices. Monodromy matrices can be obtained by considering the different representations of the quadratic algebras which have the same type of defining relations as monodromy matrices have. In this case one can use the generating series of the elements of this quadratic algebra as kind of the universal monodromy matrix and try to reformulate the Bethe ansatz on the universal level without specification to any concrete representations or concrete integrable model.

Such an approach to find the eigenvalues for the quantum integrable models with different boundary conditions and symmetries was elaborated in [1] using certain analytical assumptions on the structure of these eigenvalues. This method is called analytical Bethe ansatz and by construction was unable to yield an information on the structure of the corresponding eigenvectors. In case of the models with $\mathfrak{g l}_{N}$ symmetry the method to build the eigenvectors from the matrix elements 
of the monodromy matrices was designed in [15, 18-20] generalizing an approach of the hierarchical Bethe ansatz formulated in [14]. Authors of these papers used the universal monodromy which satisfies the commutation relations of the $\mathfrak{g l}_{N}$ Yangian or Borel subalgebra in the quantum affine algebra $U_{q}\left(\widehat{\mathfrak{g l}}_{N}\right)$ to construct the universal Bethe vectors of the hierarchical Bethe ansatz in terms of the matrix elements of the corresponding fundamental L-operators.

Quantum affine algebra and their rational analogs - the Yangian doubles have an alternative to the L-operator description [17] in term of currents [2,5]. For the quantum affine algebras, it was proved in [11] that the modes of Drinfeld currents coincide with Cartan-Weyl generators of these algebras constructed from the finite set of Chevalley generators. One may address the question whether it is possible to construct the universal Bethe vectors from the current generators of the quantum affine algebra which serves as symmetry for some quantum integrable models. This problem was investigated in [7] on a rather general level and it was shown that in order to build the universal Bethe vectors from Cartan-Weyl or current generators of the algebra one has to consider different types of Borel subalgebras in the quantum affine algebras. In this paper it was suggested to construct universal off-shell Bethe vectors for arbitrary quantum affine algebra as certain projections of products of the currents onto intersections of Borel subalgebras of different types. The generating parameters of the currents become after this identification the Bethe parameters. The papers $[12,16]$ contain detailed analysis of these projections for quantum affine algebra $U_{q}\left(\widehat{\mathfrak{g l}}_{N}\right)$. In particular, these projections are explicitly expressed via entries of the fundamental monodromy matrix and are identified with off-shell Bethe vectors of the nested Bethe ansatz [14].

An algebraic Bethe ansatz always uses a special vector which is annihilated by some ideal in the symmetry algebra (bare vacuum) and Bethe vectors are obtained by the application of the universal Bethe vectors to this vector. From the representation theory point of view we will call such bare vacuum a weight singular vector. The Cartan-Weyl generators have a good property: their products can be ordered in a natural way. If we are able to express the commuting integrals as well as the universal Bethe vectors in terms of these generators, we may rise the question: what is special in the universal Bethe vectors if their Bethe parameters satisfy the universal Bethe equations. In this paper we address this question for the quantum affine algebra $\bar{U}_{q}\left(\widehat{\mathfrak{g l}}_{N}\right)$. We found that the Cartan-Weyl ordering of the product of the universal transfer matrix and the universal Bethe vector produces the same Bethe vectors modulo elements of the ideal which annihilates the bare vacuum if the Bethe parameters satisfy the universal Bethe equations from [1].

All our calculations are performed on the level of generating series and the main technical trick which helps us to perform the ordering calculations is the identity (3.30) which is a particular case of more general relations between offshell Bethe vectors obtained in the paper [13] using the technique of the generating series.

The paper is composed as follows. In Section 2, all necessary statements for the different realizations of the quantum affine algebra $\bar{U}_{q}\left(\widehat{\mathfrak{g l}}_{N}\right)$ are given and the 
main assertion of the paper is formulated as the Theorem 1. Section 3 collects propositions which describe the ordering of the generating series of the CartanWeyl or current generators. There, an identity (3.30) is formulated: it is a new type of hierarchical relation between universal off-shell Bethe vectors expressed in terms of the current generators. Section 4 is devoted to the inductive proof of the main Theorem 1.

\section{Basic algebraic structures}

\section{1. $\bar{U}_{q}\left(\widehat{\mathfrak{g l}}_{N}\right)$ as a quantum double}

Let $q$ be a complex parameter not equal to zero or to a root of unity. Let $\mathrm{E}_{i j} \in$ $\operatorname{End}\left(\mathbb{C}^{N}\right)$ be a matrix with the only nonzero entry equal to 1 at the intersection of the $i$-th row and $j$-th column. Let $\mathrm{R}(u, v) \in \operatorname{End}\left(\mathbb{C}^{N} \otimes \mathbb{C}^{N}\right) \otimes \mathbb{C}[[v / u]]$,

$$
\begin{aligned}
\mathrm{R}(u, v)= & \sum_{1 \leq i \leq N} \mathrm{E}_{i i} \otimes \mathrm{E}_{i i}+\frac{u-v}{q u-q^{-1} v} \sum_{1 \leq i<j \leq N}\left(\mathrm{E}_{i i} \otimes \mathrm{E}_{j j}+\mathrm{E}_{j j} \otimes \mathrm{E}_{i i}\right) \\
& +\frac{q-q^{-1}}{q u-q^{-1} v} \sum_{1 \leq i<j \leq N}\left(u \mathrm{E}_{i j} \otimes \mathrm{E}_{j i}+v \mathrm{E}_{j i} \otimes \mathrm{E}_{i j}\right)
\end{aligned}
$$

be a trigonometric R-matrix associated with the vector representation of $\mathfrak{g l}_{N}$.

We will consider an associative algebra $\bar{U}_{q}\left(\widehat{\mathfrak{g l}}_{N}\right)$ with unit as a quantum double [4] of its Borel subalgebra generated by the modes $\mathrm{L}_{i, j}^{+}[k], k \geq 0,1 \leq$ $i, j \leq N$ of the L-operators $\mathrm{L}^{+}(z)=\sum_{k=0}^{\infty} \sum_{i, j=1}^{N} \mathrm{E}_{i j} \otimes \mathrm{L}_{i, j}^{+}[k] z^{-k}, \mathrm{~L}_{j, i}^{+}[0]=0$, $1 \leq i<j \leq N$ subject to the relations

$$
\mathrm{R}(u, v) \cdot\left(\mathrm{L}^{+}(u) \otimes \mathbf{1}\right) \cdot\left(\mathbf{1} \otimes \mathrm{L}^{+}(v)\right)=\left(\mathbf{1} \otimes \mathrm{L}^{+}(v)\right) \cdot\left(\mathrm{L}^{+}(u) \otimes \mathbf{1}\right) \cdot \mathrm{R}(u, v)
$$

with a standard coproduct

$$
\Delta\left(\mathrm{L}_{i, j}^{+}(u)\right)=\sum_{k=1}^{N} \mathrm{~L}_{k, j}^{+}(u) \otimes \mathrm{L}_{i, k}^{+}(u) .
$$

We denote this subalgebra $U_{q}\left(\mathfrak{b}^{+}\right) \subset \bar{U}_{q}\left(\widehat{\mathfrak{g l}}_{N}\right)$ and call it a standard Borel subalgebra of $\bar{U}_{q}\left(\widehat{\mathfrak{g l}}_{N}\right)$. In (2.2) $\mathbf{1}=\sum_{i=1}^{N} \mathrm{E}_{i i}$.

According to the general theory [4] the whole algebra $\bar{U}_{q}\left(\widehat{\mathfrak{g l}}_{N}\right)$ is generated by the modes of the L-operator $\mathrm{L}^{+}(z)$ and by the modes of the dual L-operator $\mathrm{L}^{-}(z)=\sum_{k=0}^{-\infty} \sum_{i, j=1}^{N} \mathrm{E}_{i j} \otimes \mathrm{L}_{i, j}^{-}[k] z^{-k}, \mathrm{~L}_{i, j}^{-}[0]=0,1 \leq i<j \leq N$. The dual Borel subalgebra $U_{q}\left(\mathfrak{b}^{-}\right)$has the same algebraic and coalgebraic properties (2.2) and $(2.3)$ with $\mathrm{L}^{+}(z)$ replaced by $\mathrm{L}^{-}(z)$ everywhere. The commutation relation between opposite Borel subalgebras

$$
\mathrm{R}(u, v) \cdot\left(\mathrm{L}^{+}(u) \otimes \mathbf{1}\right) \cdot\left(\mathbf{1} \otimes \mathrm{L}^{-}(v)\right)=\left(\mathbf{1} \otimes \mathrm{L}^{-}(v)\right) \cdot\left(\mathrm{L}^{+}(u) \otimes \mathbf{1}\right) \cdot \mathrm{R}(u, v),
$$

can be calculated using the non-degenerated pairing between these subalgebras. 
The standard description of the quantum affine algebra $U_{q}\left(\widehat{\mathfrak{g l}}_{N}\right)$ with dropped gradation element and at vanishing central element can be obtained from the algebra $\bar{U}_{q}\left(\widehat{\mathfrak{g l}}_{N}\right)$ by imposing one more relation

$$
\mathrm{L}_{i, i}^{+}[0] \mathrm{L}_{i, i}^{-}[0]=1 \quad i=1, \ldots, N
$$

Here we shall not assume this restriction. We shall require only invertibility of the zero modes of the diagonal matrix elements of L-operators.

\subsection{Current realization of $\bar{U}_{q}\left(\widehat{\mathfrak{g l}}_{N}\right)$}

To obtain the current realization of the algebra $\bar{U}_{q}\left(\widehat{\mathfrak{g l}}_{N}\right)[5]$ one has to introduce, according to [2], the Gauss coordinates of the L-operators. There are two different ways of introducing Gauss decompositions of L-operators. Each of these two possibilities leads to hierarchical relations for the universal Bethe vectors given by the hierarchical Bethe ansatz. One type of hierarchy occurs when the smaller algebra L-operator is embedded in the upper-left corner of the bigger algebra L-operator. The second type corresponds to the embedding in the down-right corner $[16,20]$. In this paper we will use the latter embedding. Then, Gauss coordinates $\mathrm{F}_{b, a}^{ \pm}(t)$, $\mathrm{E}_{a, b}^{ \pm}(t), b>a$ and $k_{c}^{ \pm}(t)$ are given by the decomposition:

$$
\mathrm{L}^{ \pm}(z)=\left(\mathbf{1}+\sum_{a<b}^{N} \mathrm{~F}_{b, a}^{ \pm}(z) \mathrm{E}_{a b}\right) \cdot\left(\sum_{a=1}^{N} k_{a}^{ \pm}(z) \mathrm{E}_{a a}\right) \cdot\left(\mathbf{1}+\sum_{a<b}^{N} \mathrm{E}_{a, b}^{ \pm}(z) \mathrm{E}_{b a}\right)
$$

that is to say

$$
\begin{aligned}
& \mathrm{L}_{a, b}^{ \pm}(t)=\mathrm{F}_{b, a}^{ \pm}(t) k_{b}^{+}(t)+\sum_{b<m \leq N} \mathrm{~F}_{m, a}^{ \pm}(t) k_{m}^{+}(t) \mathrm{E}_{b, m}^{ \pm}(t), \quad a<b, \\
& \mathrm{~L}_{b, b}^{ \pm}(t)=k_{b}^{ \pm}(t)+\sum_{b<m \leq N} \mathrm{~F}_{m, b}^{ \pm}(t) k_{m}^{ \pm}(t) \mathrm{E}_{b, m}^{ \pm}(t), \\
& \mathrm{L}_{a, b}^{ \pm}(t)=k_{a}^{ \pm}(t) \mathrm{E}_{b, a}^{ \pm}(t)+\sum_{a<m \leq N} \mathrm{~F}_{m, a}^{ \pm}(t) k_{m}^{ \pm}(t) \mathrm{E}_{b, m}^{ \pm}(t), \quad a>b .
\end{aligned}
$$

Considering the linear combinations of the Gauss coordinates

$$
F_{i}(t)=\mathrm{F}_{i+1, i}^{+}(t)-\mathrm{F}_{i+1, i}^{-}(t), \quad E_{i}(t)=\mathrm{E}_{i, i+1}^{+}(t)-\mathrm{E}_{i, i+1}^{-}(t)
$$


and diagonal Gauss coordinates $k_{i}^{ \pm}(t)$ one can obtain the defining relations $[2,5]$ :

$$
\begin{aligned}
\left(q^{-1} z-q w\right) E_{i}(z) E_{i}(w) & =E_{i}(w) E_{i}(z)\left(q z-q^{-1} w\right), \\
(z-w) E_{i}(z) E_{i+1}(w) & =E_{i+1}(w) E_{i}(z)\left(q^{-1} z-q w\right), \\
k_{i}^{ \pm}(z) E_{i}(w)\left(k_{i}^{ \pm}(z)\right)^{-1} & =\frac{z-w}{q^{-1} z-q w} E_{i}(w), \\
k_{i+1}^{ \pm}(z) E_{i}(w)\left(k_{i+1}^{ \pm}(z)\right)^{-1} & =\frac{z-w}{q z-q^{-1} w} E_{i}(w), \\
k_{i}^{ \pm}(z) E_{j}(w)\left(k_{i}^{ \pm}(z)\right)^{-1} & =E_{j}(w), \quad \text { if } \quad i \neq j, j+1, \\
\left(q z-q^{-1} w\right) F_{i}(z) F_{i}(w) & =F_{i}(w) F_{i}(z)\left(q^{-1} z-q w\right), \\
\left(q^{-1} z-q w\right) F_{i}(z) F_{i+1}(w) & =F_{i+1}(w) F_{i}(z)(z-w), \\
k_{i}^{ \pm}(z) F_{i}(w)\left(k_{i}^{ \pm}(z)\right)^{-1} & =\frac{q^{-1} z-q w}{z-w} F_{i}(w), \\
k_{i+1}^{ \pm}(z) F_{i}(w)\left(k_{i+1}^{ \pm}(z)\right)^{-1} & =\frac{q z-q^{-1} w}{z-w} F_{i}(w), \\
k_{i}^{ \pm}(z) F_{j}(w)\left(k_{i}^{ \pm}(z)\right)^{-1} & =F_{j}(w), \quad \text { if } \quad i \neq j, j+1, \\
{\left[E_{i}(z), F_{j}(w)\right] } & =\delta_{i, j} \delta(z / w)\left(q-q^{-1}\right)\left(k_{i}^{+}(z) / k_{i+1}^{+}(z)-k_{i}^{-}(w) / k_{i+1}^{-}(w)\right)
\end{aligned}
$$

and the Serre relations for the currents $E_{i}(z)$ and $F_{i}(z)$

$$
\begin{aligned}
\operatorname{Sym}_{z_{1}, z_{2}}\left(E_{i}\left(z_{1}\right) E_{i}\left(z_{2}\right) E_{i \pm 1}(w)\right. & -\left(q+q^{-1}\right) E_{i}\left(z_{1}\right) E_{i \pm 1}(w) E_{i}\left(z_{2}\right) \\
& \left.+E_{i \pm 1}(w) E_{i}\left(z_{1}\right) E_{i}\left(z_{2}\right)\right)=0 \\
\operatorname{Sym}_{z_{1}, z_{2}}\left(F_{i}\left(z_{1}\right) F_{i}\left(z_{2}\right) F_{i \pm 1}(w)\right. & -\left(q+q^{-1}\right) F_{i}\left(z_{1}\right) F_{i \pm 1}(w) F_{i}\left(z_{2}\right) \\
& \left.+F_{i \pm 1}(w) F_{i}\left(z_{1}\right) F_{i}\left(z_{2}\right)\right)=0 .
\end{aligned}
$$

The generating series $F_{i}(z), E_{i}(z)$ and $k_{j}^{ \pm}(z)$ are called total and Cartan currents respectively. Formulae (2.11) and (2.12) should be considered as formal series identities describing an infinite set of relations between modes of the currents. The symbol $\delta(z)$ entering these relations is the formal series $\sum_{n \in \mathbb{Z}} z^{n}$.

The algebra $\bar{U}_{q}\left(\widehat{\mathfrak{g l}}_{N}\right)$ in its current realization can be also obtained in the framework of the quantum double construction choosing another type of the Borel subalgebra. It can be constructed as the quantum double from the subalgebra $U_{F}$ generated by the modes of the currents $F_{i}[n], k_{j}^{+}[m], i=1, \ldots, N-1, j=1, \ldots, N$, $n \in \mathbb{Z}$ and $m \geq 0$. One may easily see from the commutation relations (2.11) that this is an subalgebra in $\bar{U}_{q}\left(\widehat{\mathfrak{g l}}_{N}\right)$, but for the quantum double construction one has to choose for this subalgebra a coalgebraic structure different from (2.3) [5]:

$$
\begin{aligned}
& \Delta^{(D)}\left(F_{i}(z)\right)=1 \otimes F_{i}(z)+F_{i}(z) \otimes k_{i}^{+}(z)\left(k_{i+1}^{+}(z)\right)^{-1}, \\
& \Delta^{(D)}\left(k_{i}^{+}(z)\right)=k_{i}^{+}(z) \otimes k_{i}^{+}(z) .
\end{aligned}
$$

We call $U_{F}$ a current Borel subalgebra of $\bar{U}_{q}\left(\widehat{\mathfrak{g l}}_{N}\right)$. 
The dual current Borel subalgebra $U_{E} \subset U_{q}\left(\widehat{\mathfrak{g l}}_{N}\right)$ is generated by modes of the currents $E_{i}[n], k_{j}^{-}[-m], i=1, \ldots, N-1, j=1, \ldots, N, n \in \mathbb{Z}$ and $m \geq 0$ with coalgebraic structure

$$
\begin{aligned}
& \Delta^{(D)}\left(E_{i}(z)\right)=E_{i}(z) \otimes 1+k_{i}^{-}(z)\left(k_{i+1}^{-}(z)\right)^{-1} \otimes E_{i}(z), \\
& \Delta^{(D)}\left(k_{i}^{-}(z)\right)=k_{i}^{-}(z) \otimes k_{i}^{-}(z) .
\end{aligned}
$$

In the L-operator formulation of $\bar{U}_{q}\left(\widehat{\mathfrak{g l}}_{N}\right)$ we do not use the restriction (2.5). In the current realization of the same algebra we shall not assume the relations

$$
k_{i}^{+}[0] k_{i}^{-}[0]=1, \quad i=1, \ldots, N .
$$

The latter relations are standard in the realization of the quantum affine algebra $U_{q}\left(\widehat{\mathfrak{g l}}_{N}\right)$ through a quantum double construction [5]. This realization of the quantum affine algebra $U_{q}\left(\widehat{\mathfrak{g l}}_{N}\right)$ can be obtained from $\bar{U}_{q}\left(\widehat{\mathfrak{g l}}_{N}\right)$ by imposing the relation (2.14).

\subsection{Cartan-Weyl generators of $\bar{U}_{q}\left(\widehat{\mathfrak{g l}}_{N}\right)$}

The current realization of $\bar{U}_{q}\left(\widehat{\mathfrak{g l}}_{N}\right)$ uses currents corresponding to the simple roots of $\mathfrak{g l}_{N}$ only. The modes of these currents can be identified with a part of the Cartan-Weyl generators of this quantum affine algebra. Instead of the rest CartanWeyl generators we will use the generating series introduced for the first time in [3] where they were called the composed currents.

Denote by $\bar{U}_{F}$ an extension of the current Borel subalgebra $U_{F}$ formed by the linear combinations of series, given as infinite sums of monomials $a_{i_{1}}\left[n_{1}\right] \cdots a_{i_{k}}\left[n_{k}\right]$ with $n_{1} \leq \cdots \leq n_{k}$, and $n_{1}+\cdots+n_{k}$ fixed, where $a_{i_{l}}\left[n_{l}\right]$ is either $F_{i_{l}}\left[n_{l}\right]$ or $k_{i_{l}}^{+}\left[n_{l}\right]$. Analogously, denote by $\bar{U}_{E}$ an extension of the dual current Borel subalgebra $U_{E}$ formed by the linear combinations of series, given as infinite sums of monomials $b_{i_{1}}\left[n_{1}\right] \cdots b_{i_{k}}\left[n_{k}\right]$ with $n_{1} \leq \cdots \leq n_{k}$, and $n_{1}+\cdots+n_{k}$ fixed, where $b_{i_{l}}\left[n_{l}\right]$ is either $E_{i_{l}}\left[n_{l}\right]$ or $k_{i_{l}}^{-}\left[n_{l}\right]$

First we define the composed currents $F_{j, i}(t), i<j$ as the series with coefficients in $\bar{U}_{F}$. The definition of the composed currents may be written in the analytical form

$$
F_{j, i}(t)=-\operatorname{res}_{w=t} F_{j, a}(t) F_{a, i}(w) \frac{d w}{w}=\operatorname{res}_{w=t} F_{j, a}(w) F_{a, i}(t) \frac{d w}{w}
$$

for any $a=i+1, \ldots, j-1$. It is equivalent to the relation

$$
\begin{aligned}
& F_{j, i}(t)=\oint F_{j, a}(t) F_{a, i}(w) \frac{d w}{w}-\oint \frac{q^{-1}-q t / w}{1-t / w} F_{a, i}(w) F_{j, a}(t) \frac{d w}{w}, \\
& F_{j, i}(t)=\oint F_{j, a}(w) F_{a, i}(t) \frac{d w}{w}-\oint \frac{q^{-1}-q w / t}{1-w / t} F_{a, i}(t) F_{j, a}(w) \frac{d w}{w} .
\end{aligned}
$$

In $(2.16)$ we set $\oint \frac{d w}{w} g(w)=g_{0}$ for any formal series $g(w)=\sum_{n \in \mathbb{Z}} g_{n} z^{-n}$. 
Using the relations $(2.11)$ on $F_{i}(t)$ we can calculate the residues in $(2.15)$ and obtain the following expressions for $F_{j, i}(t), i<j$ :

$$
F_{j, i}(t)=\left(q-q^{-1}\right)^{j-i-1} F_{i}(t) F_{i+1}(t) \cdots F_{j-1}(t) .
$$

For example, $F_{i+1, i}(t)=F_{i}(t)$, and $F_{i+2, i}(t)=\left(q-q^{-1}\right) F_{i}(t) F_{i+1}(t)$. Formulas (2.17) prove the consistency of the defining relations for the composed currents (2.15) or (2.16), since they yield to the same answers for all possible values $i<$ $a<j$.

Calculating formal integrals in (2.16) we obtain the following presentation for the composed currents:

$$
\begin{aligned}
& F_{j, i}(t)=F_{j, a}(t) F_{a, i}[0]-q^{-1} F_{a, i}[0] F_{j, a}(t)+\left(q-q^{-1}\right) \sum_{k<0} F_{a, i}[k] F_{j, a}(t) t^{-k}, \\
& F_{j, i}(t)=F_{j, a}[0] F_{a, i}(t)-q F_{a, i}(t) F_{j, a}[0]+\left(q-q^{-1}\right) \sum_{k \geq 0} F_{a, i}(t) F_{j, a}[k] t^{-k} .
\end{aligned}
$$

Composed currents $E_{i, j}(t)$ are defined analogously as the series with coefficients which belong to the completed current Borel subalgebra $\bar{U}_{E}$.

The fact that current generators for the quantum affine algebras form the part of the Cartan-Weyl basis in these algebras was proved in [11]. There exists a natural ordering in the Cartan-Weyl basis. If the generator $e_{\gamma}$ corresponds to a positive root $\gamma=\alpha+\beta$, where $\alpha$ and $\beta$ are the roots, then these generators are ordered either in a way $e_{\alpha} \prec e_{\gamma} \prec e_{\beta}$ or in the way $e_{\beta} \prec e_{\gamma} \prec e_{\alpha}$. An important property of the Cartan-Weyl basis of a Borel subalgebra is that the $q$-commutator of any two generators from this subalgebra, say $e_{\alpha}$ and $e_{\beta}$, is a linear combination of monomials which contain only the products of generator $e_{\gamma_{i}}$ which are 'between' $e_{\alpha}$ and $e_{\beta}: e_{\alpha} \prec e_{\gamma_{i}} \prec e_{\beta}$ or $e_{\alpha} \succ e_{\gamma_{i}} \succ e_{\beta}$. An important application of this property is that using it one can order arbitrary monomials of the generators.

The ordering on the Borel subalgebra can be extended to the ordering of the whole set of Cartan-Weyl generators corresponding to the positive and negative roots such that the same ordering property is valid. This ordering is called 'circular' or 'convex' and it allows to order arbitrary monomials in the whole algebra [7]. For the goals of our paper we will need the following specialization of this circular ordering of the current generators in the algebra $\bar{U}_{q}\left(\widehat{\mathfrak{g l}}_{N}\right)$.

Define the intersections of the different type Borel subalgebras in $\bar{U}_{q}\left(\widehat{\mathfrak{g l}}_{N}\right)$ :

$$
\begin{array}{lll}
U_{E}^{-}=U_{E} \cap U_{q}\left(\mathfrak{b}^{-}\right), & U_{f}^{-}=U_{q}\left(\mathfrak{b}^{-}\right) \cap U_{F}, \\
U_{F}^{+}=U_{F} \cap U_{q}\left(\mathfrak{b}^{+}\right), & U_{e}^{+}=U_{q}\left(\mathfrak{b}^{+}\right) \cap U_{E} .
\end{array}
$$

Let $U_{f}$ and $U_{e}$ be subalgebras of $\bar{U}_{q}\left(\widehat{\mathfrak{g l}}_{N}\right)$ formed by the modes of the currents $F_{i}(z)$ and $E_{i}(z)$ respectively. Subalgebras $U_{E}^{-}$and $U_{F}^{+}$can be also decomposed into subalgebras $U_{e}^{-}, U_{k}^{-}$and $U_{f}^{+}, U_{k}^{+}$, where

$$
U_{e}^{-}=U_{e} \cap U_{E}^{-}, \quad U_{f}^{+}=U_{f} \cap U_{F}^{+}
$$




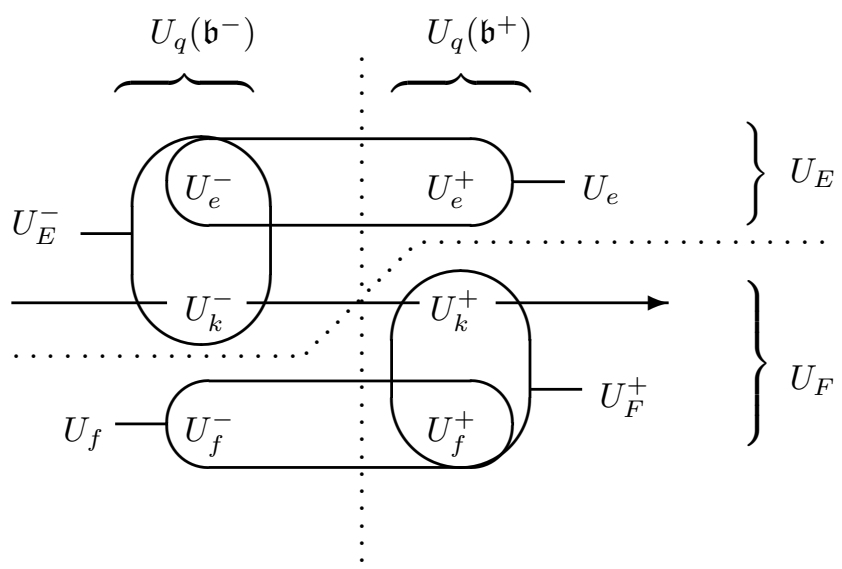

FiguRE 1. Subalgebras of $\bar{U}_{q}\left(\widehat{\mathfrak{g l}}_{N}\right)$. Vertical dotted line separates standard Borel subalgebras. Horizontal dotted line separates current Borel subalgebras. Horizontal solid axis shows increasing of the modes of the current generators. Ovals signify different subalgebras in the standard and current Borel subalgebras of $\bar{U}_{q}\left(\widehat{\mathfrak{g l}}_{N}\right)$.

and $U_{k}^{ \pm}$are defined by the isomorphisms

$$
U_{f}^{+} \otimes U_{k}^{+} \rightarrow U_{F}^{+}=U_{f}^{+} \cdot U_{k}^{+}, \quad U_{e}^{-} \otimes U_{k}^{-} \rightarrow U_{E}^{-}=U_{e}^{-} \cdot U_{k}^{-} .
$$

Different relations between these subalgebras are represented schematically in Figure 1.

We fix a 'circular' ordering ' $\prec$ ' on the generators of $\bar{U}_{q}\left(\widehat{\mathfrak{g l}}_{N}\right)$ (see [7]), such that:

$$
\cdots \prec U_{k}^{-} \prec U_{f}^{-} \prec U_{f}^{+} \prec U_{k}^{+} \prec U_{e}^{+} \prec U_{e}^{-} \prec U_{k}^{-} \prec \cdots .
$$

Definition 2.1. We will call an element $W \in \bar{U}_{q}\left(\widehat{\mathfrak{g l}}_{N}\right)$ normal ordered and denote it as : $W$ : if it is presented as the linear combinations of the products the elements $W_{1} \cdot W_{2} \cdot W_{3} \cdot W_{4} \cdot W_{5} \cdot W_{6}$ such that

$$
\begin{aligned}
W_{1} \in U_{f}^{-}, \quad W_{2} \in U_{f}^{+}, \quad W_{3} \in U_{k}^{+}, \quad W_{4} \in U_{e}^{+}, \\
\\
W_{5} \in U_{e}^{-}, \quad W_{6} \in U_{k}^{-} .
\end{aligned}
$$

We may consider standard Borel subalgebras as ordered with respect to the circular ordering $(2.22)$ :

$$
U_{q}\left(\mathfrak{b}^{-}\right)=U_{e}^{-} \cdot U_{k}^{-} \cdot U_{f}^{-}, \quad U_{q}\left(\mathfrak{b}^{+}\right)=U_{f}^{+} \cdot U_{k}^{+} \cdot U_{e}^{+} .
$$

Analogous statement is valid for the current Borel subalgebras:

$$
U_{F}=U_{f}^{-} \cdot U_{f}^{+} \cdot U_{k}^{+}, \quad U_{E}=U_{e}^{+} \cdot U_{e}^{-} \cdot U_{k}^{-} .
$$




\subsection{Projections and universal off-shell Bethe vectors}

It was proved in [10] that the subalgebras $U_{f}^{-}$and $U_{F}^{+}$are coideals with respect to the Drinfeld coproduct $(2.13)$

$$
\Delta^{(D)}\left(U_{F}^{+}\right) \subset \bar{U}_{q}\left(\widehat{\mathfrak{g l}}_{N}\right) \otimes U_{F}^{+}, \quad \Delta^{(D)}\left(U_{f}^{-}\right) \subset U_{f}^{-} \otimes \bar{U}_{q}\left(\widehat{\mathfrak{g l}}_{N}\right),
$$

and that the multiplication $m$ in $\bar{U}_{q}\left(\widehat{\mathfrak{g l}}_{N}\right)$ induces an isomorphism of vector spaces

$$
m: U_{f}^{-} \otimes U_{F}^{+} \rightarrow U_{F} .
$$

According to the general theory presented in [7] we define projection operators $P_{f}^{+}: U_{F} \subset \bar{U}_{q}\left(\widehat{\mathfrak{g l}}_{N}\right) \rightarrow U_{F}^{+}$and $P_{f}^{-}: U_{F} \subset \bar{U}_{q}\left(\widehat{\mathfrak{g l}}_{N}\right) \rightarrow U_{f}^{-}$by the prescriptions

$$
\begin{gathered}
P_{f}^{+}\left(f_{-} f_{+}\right)=\varepsilon\left(f_{-}\right) f_{+}, \quad P_{f}^{-}\left(f_{-} f_{+}\right)=f_{-} \varepsilon\left(f_{+}\right), \\
\text {for any } f_{-} \in U_{f}^{-}, \quad f_{+} \in U_{F}^{+} .
\end{gathered}
$$

It was also proved in [7] that

(1) projections $(2.24)$ can be extended to the algebra $\bar{U}_{F}$;

(2) for any $f \in \bar{U}_{F}$ with $\Delta^{(D)}(f)=\sum_{i} f_{i}^{\prime} \otimes f_{i}^{\prime \prime}$ we have

$$
f=\sum_{i} P_{f}^{-}\left(f_{i}^{\prime \prime}\right) \cdot P_{f}^{+}\left(f_{i}^{\prime}\right) .
$$

Analogously, we may define projections of the dual current Borel subalgebras $U_{E}$ : $P_{e}^{-}: U_{E} \rightarrow U_{E}^{-}$and $P_{e}^{+}: U_{E} \rightarrow U_{e}^{+}$.

Let $\bar{n}=\left\{n_{1}, n_{2}, \ldots, n_{N-2}, n_{N-1}\right\}$ be a set of non-negative integers. Denote by $\bar{t}_{[\bar{n}]}$ the set of formal variables:

$$
\bar{t}_{[\bar{n}]}=\left\{t_{1}^{1}, \ldots, t_{n_{1}}^{1} ; t_{1}^{2}, \ldots, t_{n_{2}}^{2} ; \ldots \ldots ; t_{1}^{N-2}, \ldots, t_{n_{N-2}}^{N-2} ; t_{1}^{N-1}, \ldots, t_{n_{N-1}}^{N-1}\right\} .
$$

The variable $t_{k}^{a}$ is of type $a$. If $n_{a}=0$ for some $a$, then the variables of type $a$ are absent in the set $(2.26)$. Denote by $\mathcal{W}_{N}\left(\bar{t}_{[\bar{n}]}\right)$ the universal weight function associated with the set of variables $(2.26):^{1}$

$$
\mathcal{W}_{N}\left(\bar{t}_{[\bar{n}]}\right)=P_{f}^{+}\left(F_{N-1}\left(t_{n_{N-1}}^{N-1}\right) \cdots F_{N-1}\left(t_{1}^{N-1}\right) \quad \cdots \quad F_{1}\left(t_{n_{1}}^{1}\right) \cdots F_{1}\left(t_{1}^{1}\right)\right) .
$$

By definition, the universal weight function (2.27) is a formal Laurent series of all variables $1 / t_{i}^{a}$, the ratios $t_{k}^{b} / t_{l}^{c}$ with $b<c$ and $t_{i}^{a} / t_{j}^{a}$ with $i<j$ taking values in the completion $\bar{U}_{F}$ :

$$
\mathcal{W}_{N}\left(\bar{t}_{[\bar{n}]}\right) \in \mathrm{A}\left[\left[\left\{1 / t_{m}^{a}, t_{k}^{b} /\left.t_{l}^{c}\right|_{b<c}, t_{i}^{d} /\left.t_{j}^{d}\right|_{i<j}\right\}\right]\right]\left[\left\{t_{m}^{e}\right\}\right], \quad \mathrm{A}=\bar{U}_{F} .
$$

The calculations made in [12] show that it is indeed a formal power series in the variables $1 / t_{i}^{a}, t_{k}^{b} / t_{l}^{c}$ with $b<c$ and $t_{i}^{a} / t_{j}^{a}$ with $i<j$ taking values in $\bar{U}_{F}$.

It was proved in $[7,10]$ that this projection satisfies the comultiplication properties of the off-shell Bethe vectors of the hierarchical Bethe ansatz [14]. In [9], a method of calculation of the projection (2.27) based on the ordering

${ }^{1}$ In contrast to the paper [13], here we will not normalize the universal weight function by the product of factorials. 
property $(2.25)$ was proposed. Then in $[12,16]$, this method was applied to the quantum affine algebra $U_{q}\left(\widehat{\mathfrak{g l}}_{N}\right)$. It was proved there that the calculation of the projection (2.27) produces the same hierarchical relations for the universal off-shell Bethe vectors as were found in [20] using combinatorial methods in the framework of the standard hierarchical Bethe ansatz.

We call a vector $v$ a weight singular vector if it is annihilated by any positive mode $E_{i}[n], i=1, \ldots, N-1, n>0$ and is an eigenvector for $k_{i}^{+}(t), i=1, \ldots, N$

$$
\mathrm{E}_{i, i+1}^{+}(t) \cdot v=0, \quad k_{i}^{+}(t) \cdot v=\lambda_{i}(t) v,
$$

where $\lambda_{i}(t)$ is a meromorphic function, decomposed as a power series in $t^{-1}$. The $L$-operator (2.6), acting on a weight singular vector $v$, becomes upper-triangular

$$
\mathrm{L}_{i j}^{+}(t) v=0, \quad i>j, \quad \mathrm{~L}_{i i}^{+}(t) v=\lambda_{i}(t) v, \quad i=1, \ldots, N .
$$

Definition 2.2. We note $J$ the left ideal of $U_{q}\left(\mathfrak{b}^{+}\right)$, generated by all elements of the form $U_{q}\left(\mathfrak{b}^{+}\right) \cdot E_{i}[n], i=1, \ldots, N-1, n>0$ (equivalently, by all modes of $\left.U_{q}\left(\mathfrak{b}^{+}\right) \cdot \mathrm{E}_{i, j}^{+}(t), 1 \leq i<j \leq N\right)$.

Equalities in $U_{q}\left(\mathfrak{b}^{+}\right)$modulo element from the ideal $J$ we denote by the symbol ' $\sim_{J}$ '.

It is clear that $W \cdot v=0$ for any element $W \in J$ and arbitrary weight singular vector $v$.

We call a (universal) transfer matrix the trace of L-operator

$$
\mathcal{T}_{N}(t)=\sum_{i=1}^{N} \mathrm{~L}_{i, i}^{+}(t)=\sum_{i=1}^{N}\left(k_{i}^{+}(t)+\sum_{j=i+1}^{N} \mathrm{~F}_{j, i}^{+}(t) k_{j}^{+}(t) \mathrm{E}_{i, j}^{+}(t)\right)
$$

The Gauss coordinates $\mathrm{F}_{j, i}^{+}(t), \mathrm{E}_{i, j}^{+}(t)$ coincide with the projections of the corresponding composed currents and can be expressed through modes of the currents from subalgebras $U_{f}^{+}$and $U_{e}^{+}$(see section 3.1). Note that the presentation (2.30) of the transfer matrix $\mathcal{T}_{N}(t)$ is normal ordered according to the circular ordering $(2.22)$ and $\mathcal{T}_{N}(t) \sim_{J} \sum_{i=1}^{N} k_{i}^{+}(t)$.

Definition 2.3. Let $\mathcal{B}$ be the left ideal of $U_{q}\left(\mathfrak{b}^{+}\right)$, generated by all elements of the form $U_{F}^{+} \cdot \mathrm{b}$, where $\mathrm{b}$ are the modes of the series

$\mathbf{b}_{j}^{i}\left(\bar{t}_{[\bar{n}]}\right)=\frac{k_{i}^{+}\left(t_{j}^{i}\right)}{k_{i+1}^{+}\left(t_{j}^{i}\right)}-\prod_{\substack{m=1 \\ m \neq j}}^{n_{i}} \frac{q-q^{-1} t_{m}^{i} / t_{j}^{i}}{q^{-1}-q t_{m}^{i} / t_{j}^{i}} \prod_{m=1}^{n_{i-1}} \frac{1-t_{m}^{i-1} / t_{j}^{i}}{q-q^{-1} t_{m}^{i-1} / t_{j}^{i}} \prod_{m=1}^{n_{i+1}} \frac{q^{-1}-q t_{m}^{i+1} / t_{j}^{i}}{1-t_{m}^{i+1} / t_{j}^{i}}$.

Here $i=1, \ldots, N-1, j=1, \ldots, n_{i}$. Equalities in $U_{q}\left(\mathfrak{b}^{+}\right)$modulo elements from the ideal $\mathcal{B}$ we denote by the symbol ' $\sim_{\mathcal{B}}$ '. We call this ideal the Bethe ideal and equations for the set of the Bethe parameters $\left\{t_{j}^{i}\right\}$

$$
\mathrm{b}_{j}^{i}\left(\bar{t}_{[\bar{n}]}\right)=0, \quad i=1, \ldots, N-1, \quad j=1, \ldots, n_{i},
$$

the universal Bethe equations. 
The main statement of this paper is

Theorem 1. A formal series identity with coefficients in $U_{q}\left(\mathfrak{b}^{+}\right)$

$$
\mathcal{T}_{N}(t) \cdot \mathcal{W}_{N}\left(\bar{t}_{[\bar{n}]}\right)-\mathcal{W}_{N}\left(\bar{t}_{[\bar{n}]}\right) \cdot \tau_{N}\left(t ; \bar{t}_{[\bar{n}]}\right) \sim_{J, \mathcal{B}} 0
$$

is valid modulo elements from the ideals $J$ and $\mathcal{B}$. Here

$$
\tau_{N}\left(t ; \bar{t}_{[\bar{n}]}\right)=\sum_{i=1}^{N} k_{i}^{+}(t) \prod_{j=1}^{n_{i-1}} \frac{q-q^{-1} t_{j}^{i-1} / t}{1-t_{j}^{i-1} / t} \prod_{j=1}^{n_{i}} \frac{q^{-1}-q t_{j}^{i} / t}{1-t_{j}^{i} / t}
$$

is an eigenvalue of the universal transfer matrix.

One may understand the universal Bethe equations (2.32) as a relation between Bethe parameters $\bar{t}_{[\bar{n}]}$ with coefficients in the commutative subalgebra $U_{k}^{+} \subset$ $U_{q}\left(\mathfrak{b}^{+}\right)$generated by the modes of the commuting Cartan currents $k_{i}^{+}(t)$. In a representation with a generating singular vector $v$ these modes are being replaced by the corresponding eigenvalues of $v$.

Proving the statement of Theorem 1 we will try to present the product $\mathcal{T}_{N}(t) \cdot \mathcal{W}_{N}\left(\bar{t}_{[\bar{n}]}\right)$ in the normal ordered form according to the ordering given in Definition 2.1. After performing this ordering we will observe that subtraction of the ordered product $\mathcal{W}_{N}\left(\bar{t}_{[\bar{n}]}\right) \cdot \tau_{N}\left(t ; \bar{t}_{[\bar{n}]}\right)$ results only in the terms which belong either to the ideal $J$ or to the ideal $\mathcal{B}$.

For any weight singular vector $v$, let $w_{V}^{N}\left(\bar{t}_{[\bar{n}]}\right)=\mathcal{W}_{N}\left(\bar{t}_{[\bar{n}]}\right) v$ be the weight function taking value in the $\bar{U}_{q}\left(\widehat{\mathfrak{g l}}_{N}\right)$-module $V$ generated by $v$ and

$$
\mathbf{w}_{V}^{N}\left(\bar{t}_{[\bar{n}]}\right)=\beta\left(\bar{t}_{[\bar{n}]}\right) \prod_{a=2}^{N} \prod_{\ell=1}^{n_{a-1}} \lambda_{a}\left(t_{\ell}^{a-1}\right) w_{V}^{N}\left(\bar{t}_{[\bar{n}]}\right)
$$

be the corresponding modified weight function [10]. Here

$$
\beta\left(\bar{t}_{[\bar{n}]}\right)=\prod_{a=1}^{N-1} \prod_{1 \leq \ell<\ell^{\prime} \leq n_{a}} \frac{q-q^{-1} t_{\ell}^{a} / t_{\ell^{\prime}}^{a}}{1-t_{\ell}^{a} / t_{\ell^{\prime}}^{a}} .
$$

According to $[10,12,20]$ we call the modified weight function $(2.35)$ universal offshell Bethe vector. The Theorem 1 has obvious

Corollary 2.4. Universal off-shell Bethe vector is an eigenvector of the universal transfer matrix

$$
\mathcal{T}_{N}(t) \cdot \mathbf{w}_{V}^{N}\left(\bar{t}_{[\bar{n}]}\right)=\mathbf{w}_{V}^{N}\left(\bar{t}_{[\bar{n}]}\right) \cdot\left(\sum_{i=1}^{n} \lambda_{i}(t) \prod_{j=1}^{n_{i-1}} \frac{q t-q^{-1} t_{j}^{i-1}}{t-t_{j}^{i-1}} \prod_{j=1}^{n_{i}} \frac{q^{-1} t-q t_{j}^{i}}{t-t_{j}^{i}}\right)
$$

if Bethe equations of $U_{q}\left(\widehat{\mathfrak{g l}}_{N}\right)$ hierarchical Bethe ansatz are satisfied:

$$
\frac{\lambda_{i}\left(t_{j}^{i}\right)}{\lambda_{i+1}\left(t_{j}^{i}\right)}=\prod_{m \neq j}^{n_{i}} \frac{q t_{j}^{i}-q^{-1} t_{m}^{i}}{q^{-1} t_{j}^{i}-q t_{m}^{i}} \prod_{m=1}^{n_{i-1}} \frac{t_{j}^{i}-t_{m}^{i-1}}{q t_{j}^{i}-q^{-1} t_{m}^{i-1}} \prod_{m=1}^{n_{i+1}} \frac{q^{-1} t_{j}^{i}-q t_{m}^{i+1}}{t_{j}^{i}-t_{m}^{i+1}} .
$$




\section{Proofs}

We will prove the Theorem 1 by induction over $N$. First, we check that the statement of the theorem is valid in the simplest case $N=2$. Then, assuming the correctness of the statement for the algebra $\bar{U}_{q}\left(\widehat{\mathfrak{g l}}_{N}\right)$ we will prove it for the algebra $\bar{U}_{q}\left(\widehat{\mathfrak{g l}}_{N+1}\right)$. The L-operator of the smaller algebra $\bar{U}_{q}\left(\widehat{\mathfrak{g l}}_{N}\right)$ will be embedded into right down corner of the L-operator for the bigger algebra $\bar{U}_{q}\left(\widehat{\mathfrak{g l}}_{N+1}\right)$. This embedding is in accordance with the Gauss decomposition (2.6). The main technical tool will be a special presentation of the universal weight function based on the main property of the projections (2.24). This allows to reduce the calculation of the ordering to the commutations of the Gauss coordinates and total currents, which is much simpler than the exchange relations of these coordinates with the projection (2.27).

\subsection{Relation between Gauss coordinates and the currents}

In order to perform the proof of the main theorem we need to establish a precise relation between all Gauss coordinates and the currents. This was done partially in [10] and here we repeat this calculations for the sake of completeness. Note that the paper [2] yields these relations only for currents corresponding to simple roots.

Set $S_{A}(B)=B A-q^{-1} A B$. Projections of composed currents can be defined using $q$-commutators with zero modes of the currents $F_{i}(t), i=1, \ldots, N-1$. We will call the operators $S_{F_{i}[0]} \equiv S_{i}$ the screening operators.

Proposition 3.1. We have

$$
\begin{aligned}
& P_{f}^{+}\left(F_{j, i}(t)\right)=S_{i}\left(P_{f}^{+}\left(F_{j, i+1}(t)\right)\right), \\
& P_{f}^{-}\left(F_{j, i}(t)\right)=-q S_{j-1}\left(P_{f}^{-}\left(F_{j-1, i}(t)\right)\right), \quad i<j-1 .
\end{aligned}
$$

Proof. We apply the projection $P_{f}^{+}$to both sides of the relation (2.18) with $a=$ $i+1$ and the projection $P_{f}^{-}$to both sides of the relation (2.19) with $a=j-1$. The modes $F_{i}[k]$ with $k<0$ belong to $U_{f}^{-}$and the modes $F_{j-1}[k]$ with $k \geq 0$ belong to $U_{f}^{+}$. Hence, due to formulae $(2.24)$, the projections $P_{f}^{ \pm}$both kill the semi-infinite sum in the right hand side of (2.18) and (2.19), and we get

$$
\begin{aligned}
P_{f}^{+}\left(F_{j, i}(t)\right) & =P_{f}^{+}\left(F_{j, i+1}(t) F_{i}[0]-q^{-1} F_{i}[0] F_{j, i+1}(t)\right) \\
& =P_{f}^{+}\left(S_{i}\left(F_{j, i+1}(t)\right)\right)=S_{i}\left(P_{f}^{+}\left(F_{j, i+1}(t)\right)\right)
\end{aligned}
$$

and

$$
\begin{aligned}
P_{f}^{-}\left(F_{i, j}(t)\right) & =-q P_{f}^{-}\left(F_{j-1, i}(t) F_{j, j-1}[0]-q^{-1} F_{j, j-1}[0] F_{j-1, i}(t)\right) \\
& =-q P_{f}^{-}\left(S_{j-1}\left(F_{j-1, i}(t)\right)\right)=-q S_{j-1}\left(P_{f}^{-}\left(F_{j-1, i}(t)\right)\right) .
\end{aligned}
$$

To get the last equalities we use the fact proved in [9] that the projection $P_{f}^{ \pm}$ commutes with the screening operators $S_{i}: P_{f}^{ \pm}\left(S_{i}(W)\right)=S_{i}\left(P_{f}^{ \pm}(W)\right)$ for any $W \in U_{f}$. 
The screening operators also relate the Gauss coordinates of the L-operators.

Lemma 3.2. We have

$$
\left(q-q^{-1}\right) \mathrm{F}_{j, i}^{ \pm}(t)=S_{i}\left(\mathrm{~F}_{j, i+1}^{ \pm}(t)\right), \quad i<j-1 .
$$

Proof of this lemma was given in $[10]^{2}$ and is based on the commutation relation between matrix elements $\mathrm{L}_{i, i+1}^{+}(s)$ and $\mathrm{L}_{i+1, j}^{ \pm}(t)$. One should consider the coefficients at the zero power of the spectral parameter $s$ in these relations and take into account that $\mathrm{L}_{i, i+1}^{+}[0]=F_{i}[0] k_{i+1}^{+}[0]$ and $\mathrm{L}_{i+1, i+1}^{+}[0]=k_{i+1}^{+}[0]$.

Proposition 3.1 and Lemma 3.2 imply the following

Proposition 3.3. We have

$$
P_{f}^{+}\left(F_{j, i}(t)\right)=\left(q-q^{-1}\right)^{j-i-1} \mathrm{~F}_{j, i}^{+}(t), \quad i<j-1,
$$

$$
\begin{aligned}
& P_{f}^{-}\left(F_{j, i}(t)\right) \\
= & \left(q-q^{-1}\right)^{j-i-1}\left(\mathrm{~F}_{j, i}^{-}(t)+\sum_{\ell=1}^{j-i-1}(-1)^{\ell} \sum_{j>i_{\ell}>\cdots>i_{1}>i} \mathrm{~F}_{i_{1}, i}^{-}(t) \cdots \mathrm{F}_{j, i_{\ell}}^{-}(t)\right) .
\end{aligned}
$$

Proof. First equality (3.5) was proved in [10] using induction with respect to $j-i$ from the formula $P_{f}^{+}\left(F_{i+1, i}(t)\right)=\mathrm{F}_{i+1, i}^{+}(t)[2]$. Here we shall prove (3.6). We apply the projection $P_{f}^{-}$to both sides of the relation (2.18) to obtain

$$
P_{f}^{-}\left(F_{j, i}(t)\right)=S_{i}\left(P_{f}^{-}\left(F_{j, i+1}(t)\right)\right)+\left(q-q^{-1}\right) P_{f}^{-}\left(F_{i+1, i}(t)\right) \cdot P_{f}^{-}\left(F_{j, i+1}(t)\right) .
$$

Using this relation recursively and the Lemma 3.2, we get

$$
\begin{aligned}
P_{f}^{-}\left(F_{j, i}(t)\right)+\left(q-q^{-1}\right)^{j-i-1} \mathrm{~F}_{j, i}^{-}(t) & \\
& +\sum_{\ell=i+1}^{j-1}\left(q-q^{-1}\right)^{j-\ell} \mathrm{F}_{\ell, i}^{-}(t) \cdot P_{f}^{-}\left(\mathrm{F}_{j, \ell}(t)\right)=0 .
\end{aligned}
$$

From the identity

$$
P_{f}^{-}\left(F_{i+1, i}(t)\right)=-\mathrm{F}_{i+1, i}^{-}(t)
$$

one proves that equality (3.6) is a solution of this recurrence relation, and coincide with it for $i=j-1$.

Proceeding in analogous way, we may relate the projections of the dual composed currents $P_{e}^{ \pm}\left(E_{i, j}(t)\right)$ with Gauss coordinates $\mathrm{E}_{i, j}^{ \pm}(t)$, but here we shall need only the relations between different dual Gauss coordinates or analog of the Lemma 3.2 for $\mathrm{E}_{i, j}^{ \pm}(t)$. Set $\hat{S}_{A}(B)=A B-q B A$ and denote $\hat{S}_{E_{i}[0]} \equiv \hat{S}_{i}$.

${ }^{2}$ See also the proof of the analogous Lemma 3.4 below. 
Lemma 3.4. We have

$$
\left(q-q^{-1}\right) \mathrm{E}_{i, j}^{ \pm}(t)=\hat{S}_{i}\left(\mathrm{E}_{i+1, j}^{ \pm}(t)\right), \quad i<j-1 .
$$

Proof. Let us consider the commutation relations between the following matrix elements of L-operators

$$
\begin{aligned}
& (t-s)\left[\mathrm{L}_{j, i+1}^{ \pm}(t), \mathrm{L}_{i+1, i}^{-}(s)\right] \\
& \quad=\left(q-q^{-1}\right)\left(t \mathrm{~L}_{i+1, i+1}^{-}(s) \mathrm{L}_{j, i}^{ \pm}(t)-s \mathrm{~L}_{i+1, i+1}^{ \pm}(t) \mathrm{L}_{j, i}^{-}(s)\right) \\
& \left(q t-q^{-1} s\right) \mathrm{L}_{i+1, i+1}^{-}(s) \mathrm{L}_{j, i+1}^{ \pm}(t) \\
& \quad=(t-s) \mathrm{L}_{j, i+1}^{ \pm}(t) \mathrm{L}_{i+1, i+1}^{-}(s)+\left(q-q^{-1}\right) s \mathrm{~L}_{i+1, i+1}^{ \pm}(t) \mathrm{L}_{j, i+1}^{-}(s) .
\end{aligned}
$$

Choosing the coefficients at the zero power of the spectral parameter $s$ in these relations and taking into account that $\mathrm{L}_{i+1, i}^{-}[0]=-k_{i+1}^{-}[0] E_{i}[0]$ and $\mathrm{L}_{i+1, i+1}^{-}[0]=$ $k_{i+1}^{-}[0]$ we obtain

$$
\left(q-q^{-1}\right) \mathrm{L}_{j, i}^{ \pm}(t)=E_{i}[0] \mathrm{L}_{j, i+1}^{ \pm}(t)-q \mathrm{~L}_{j, i+1}^{ \pm}(t) E_{i}[0]=\hat{S}_{i}\left(\mathrm{~L}_{j, i+1}^{ \pm}(t)\right) .
$$

In order to obtain (3.10) we shall use an explicit expression for the matrix elements of the L-operator (2.9) in terms of the Gauss coordinates. The relation (3.11) implies (3.10) for $j=N$ due to the commutativity of $k_{N}^{ \pm}(t)$ and $E_{i}[0]$ for $i=$ $1, \ldots, N-2$. Next, the relations (3.11) for $j=N-1$ and (3.10) for $j=N$ imply (3.10) for $j=N-1$ due to the commutativity of the Gauss coordinates $k_{N-1}^{ \pm}(t)$, $k_{N}^{ \pm}(t)$ and $\mathrm{F}_{N, N-1}^{ \pm}(t)$ with $E_{i}[0]$ for $i=1, \ldots, N-3$. The statement of the lemma follows by induction over $j$.

\subsection{Basic notations}

Let $\bar{l}$ and $\bar{r}$ be two collections of nonnegative integers satisfying a set of inequalities

$$
l_{a} \leq r_{a}, \quad a=1, \ldots, N-1 .
$$

Denote by $[\bar{l}, \bar{r}]$ a set of segments which contain positive integers $\left\{l_{a}+1, l_{a}+\right.$ $\left.2, \ldots, r_{a}-1, r_{a}\right\}$ including $r_{a}$ and excluding $l_{a}$. The length of each segment is equal to $r_{a}-l_{a}$.

For a given set $[\bar{l}, \bar{r}]$ of segments we denote by $\bar{t}_{[\bar{l}, \bar{r}]}$ the sets of variables

$$
\bar{t}_{[\bar{l}, \bar{r}]}=\left\{t_{l_{1}+1}^{1}, \ldots, t_{r_{1}}^{1} ; t_{l_{2}+1}^{2}, \ldots, t_{r_{2}}^{2} ; \ldots ; t_{l_{N-1}+1}^{N-1}, \ldots, t_{r_{N-1}}^{N-1}\right\} .
$$

For any $a=1, \ldots, N-1$ we denote the sets of variables corresponding to the segments $\left[l_{a}, r_{a}\right]=\left\{l_{a}+1, l_{a}+2, \ldots, r_{a}\right\}$ as $\bar{t}_{\left[l_{a}, r_{a}\right]}^{a}=\left\{t_{l_{a}+1}^{a}, \ldots, t_{r_{a}}^{a}\right\}$. All the variables in $\bar{t}_{\left[l_{a}, r_{a}\right]}^{a}$ have type $a$. For the segments $\left[l_{a}, r_{a}\right]=\left[0, n_{a}\right]$ we use the shorten notations $\bar{t}_{[\overline{0}, \bar{n}]} \equiv \bar{t}_{[\bar{n}]}$ and $\bar{t}_{\left[0, n_{a}\right]}^{a} \equiv \bar{t}_{\left[n_{a}\right]}^{a}$. 
For a collection of variables $\bar{t}_{[\bar{l}, \bar{r}]}$ we consider the ordered products of the currents

$$
\begin{aligned}
\mathcal{F}\left(\bar{t}_{[\bar{l}, \bar{r}]}\right) & =\prod_{N-1 \geq a \geq 1}\left(\prod_{r_{a} \geq \ell>l_{a}} F_{a}\left(t_{\ell}^{a}\right)\right) \\
& =F_{N-1}\left(t_{r_{N-1}}^{N-1}\right) \cdots F_{1}\left(t_{r_{1}}^{1}\right) \cdots F_{1}\left(t_{l_{1}+1}^{1}\right)
\end{aligned}
$$

where the series $F_{a}(t) \equiv F_{a+1, a}(t)$ are defined by (2.10). As particular cases, we have $\mathcal{F}\left(\bar{t}_{\left[l_{a}, r_{a}\right]}^{a}\right)=F_{a}\left(t_{r_{a}}^{a}\right) \cdots F_{a}\left(t_{l_{a}+2}^{a}\right) F_{a}\left(t_{l_{a}+1}^{a}\right)$.

The product (3.14) is a formal series over the ratios $t_{k}^{b} / t_{l}^{c}$ with $b<c$ and $t_{i}^{a} / t_{j}^{a}$ with $i<j$ taking values in the algebra $U_{F}$.

Symbol $\prod_{a}^{\longleftarrow} A_{a}$ (resp. $\prod_{a}^{\longrightarrow} A_{a}$ ) means the ordered products of noncommutative entries $A_{a}$, such that $A_{a}$ is on the right (resp. on the left) from $A_{b}$ for $b>a$ :

$$
\overleftarrow{\prod_{j \geq a \geq i}} A_{a}=A_{j} A_{j-1} \cdots A_{i+1} A_{i}, \quad \prod_{i \leq a \leq j} A_{a}=A_{i} A_{i+1} \cdots A_{j-1} A_{j}
$$

Consider the permutation group $S_{n}$ and its action on the formal series of $n$ variables defined for the elementary transpositions $\sigma_{i, i+1}$ as follows

$$
\pi\left(\sigma_{i, i+1}\right) G\left(t_{1}, \ldots, t_{i}, t_{i+1}, \ldots, t_{n}\right)=\frac{q^{-1}-q t_{i} / t_{i+1}}{q-q^{-1} t_{i} / t_{i+1}} G\left(t_{1}, \ldots, t_{i+1}, t_{i}, \ldots, t_{n}\right) .
$$

The $q$-depending factor in this formula is chosen in such a way that each product $F_{a}\left(t_{n}\right) \cdots F_{a}\left(t_{1}\right)$ is invariant under this action. Summing the action over all the group of permutations we obtain the operator $\overline{\mathrm{Sym}}_{u}=\frac{1}{n !} \sum_{\sigma \in S_{n}} \pi(\sigma)$ acting as follows ${ }^{3}$

$$
\overline{\operatorname{Sym}}_{\bar{t}} G(\bar{t})=\frac{1}{n !} \sum_{\sigma \in S_{n}} \prod_{\substack{\ell<\ell^{\prime} \\ \sigma(\ell)>\sigma\left(\ell^{\prime}\right)}} \frac{q^{-1}-q t_{\sigma\left(\ell^{\prime}\right)} / t_{\sigma(\ell)}}{q-q^{-1} t_{\sigma\left(\ell^{\prime}\right)} / t_{\sigma(\ell)}} G\left({ }^{\sigma} t\right) .
$$

The product is taken over all pairs $\left(\ell, \ell^{\prime}\right)$, such that conditions $\ell<\ell^{\prime}$ and $\sigma(\ell)>$ $\sigma\left(\ell^{\prime}\right)$ are satisfied simultaneously.

We call the operator $\overline{\operatorname{Sym}}_{u}$ a q-symmetrization. The operator $\overline{\mathrm{Sym}}_{u}$ is the group average with respect to the action $\pi$, so that

$$
\overline{\operatorname{Sym}}_{\bar{t}} \overline{\operatorname{Sym}}_{\bar{t}}(\cdot)=\overline{\operatorname{Sym}}_{\bar{t}}(\cdot) \text {. }
$$

An important property of $q$-symmetrization is the relation

$$
\overline{\operatorname{Sym}}_{\left(t_{1}, \ldots, t_{n}\right)}=\frac{s !(n-s) !}{n !} \sum_{\sigma \in S_{n}^{(s)}} \pi(\sigma) \overline{\operatorname{Sym}}_{\left(t_{1}, \ldots, t_{s}\right)} \overline{\operatorname{Sym}}_{\left(t_{s+1}, \ldots, t_{n}\right)},
$$

${ }^{3}$ Normalization of the $q$-symmetrization used here differs from the one used in the papers $[12,16]$ by the combinatorial factor $\frac{1}{n !}$. 
where $s \in[0, n]$ is fixed and the sum is taken over the subset

$$
S_{n}^{(s)}=\left\{\sigma \in S_{n} \mid \sigma(1)<\cdots<\sigma(s) ; \sigma(s+1)<\cdots<\sigma(n)\right\} .
$$

Denote by $S_{[\bar{l}, \bar{r}]}=S_{\left[l_{1}, r_{1}\right]} \times \cdots \times S_{\left[l_{N-1}, r_{N-1}\right]}$ the direct product of the groups $S_{\left[l_{a}, r_{a}\right]}$ permuting integer numbers $l_{a}+1, \ldots, r_{a}$. The $q$-symmetrization over the whole set of variables $\bar{t}_{[\bar{l}, \bar{r}]}$ is defined by the formula

$$
\begin{aligned}
& \overline{\operatorname{Sym}}_{\bar{t}_{[\bar{l}, \bar{r}]}} G\left(\bar{t}_{[\bar{l}, \bar{r}]}\right) \\
& \quad=\sum_{\sigma \in S_{[\bar{l}, \bar{r}]}} \prod_{1 \leq a \leq N-1}\left(\frac{1}{\left(r_{a}-l_{a}\right) !} \prod_{\substack{\ell<\ell^{\prime} \\
\sigma^{a}(\ell)>\sigma^{a}\left(\ell^{\prime}\right)}} \frac{q^{-1}-q t_{\sigma^{a}\left(\ell^{\prime}\right)}^{a} / t_{\sigma^{a}(\ell)}^{a}}{q-q^{-1} t_{\sigma^{a}\left(\ell^{\prime}\right)}^{a} / t_{\sigma^{a}(\ell)}^{a}}\right) G\left({ }^{\sigma} \bar{t}_{[\bar{l}, \bar{r}]}\right),
\end{aligned}
$$

where the set ${ }^{\sigma} \bar{t}_{[\bar{l}, \bar{r}]}$ is defined as

$$
\begin{aligned}
{ }^{\sigma} \bar{t}_{[\bar{l}, \bar{r}]}=\left\{t_{\sigma^{1}\left(l_{1}+1\right)}^{1}, \ldots, t_{\sigma^{1}\left(r_{1}\right)}^{1}\right. & \\
& \left.t_{\sigma^{2}\left(l_{2}+1\right)}^{2}, \ldots, t_{\sigma^{2}\left(r_{2}\right)}^{2} ; \ldots ; t_{\sigma^{N-1}\left(l_{N-1}+1\right)}^{N-1}, \ldots, t_{\sigma^{N-1}\left(r_{N-1}\right)}^{N-1}\right\} .
\end{aligned}
$$

We say that the series $G\left(\bar{t}_{[\bar{l}, \bar{r}]}\right)$ is $q$-symmetric, if it is invariant under the action $\pi$ of each group $S_{\left[l_{a}, r_{a}\right]}$ with respect to the permutations of the variables $t_{l_{a}+1}^{a}, \ldots, t_{r_{a}}$ for $a=1, \ldots, N-1$ :

$$
\overline{\operatorname{Sym}}_{\bar{t}_{[\bar{l}, \bar{r}]}} G\left(\bar{t}_{[\bar{l}, \bar{r}]}\right)=G\left(\bar{t}_{[\bar{l}, \bar{r}]}\right) .
$$

The $q$-symmetrization $G\left(\bar{t}_{[\bar{l}, \bar{r}]}\right)=\overline{\operatorname{Sym}}_{\bar{t}_{[\bar{l}, \bar{r}]}} Q\left(\bar{t}_{[\bar{l}, \bar{r}]}\right)$ of any series $Q\left(\bar{t}_{[\bar{l}, \bar{r}]}\right)$ is a $q$ symmetric series, which follows from $(3.16)$.

Let $\bar{s}=\left\{s_{1}, \ldots, s_{N-1}\right\}$ be a set of nonnegative integers satisfying $l_{a} \leq s_{a} \leq r_{a}$ for $a=1, \ldots, N-1$. The set of integers $\bar{s}$ divides the set of the variables $\bar{t}_{[\bar{l}, \bar{r}]}$ into two subsets $\bar{t}_{[\bar{s}, \bar{r}]} \cup \bar{t}_{[\bar{l}, \bar{s}]}$.

Using the property of the projections (2.25) we can present any product of the currents in a normal ordered form (in the sense of Definition 2.1):

$$
\begin{aligned}
\mathcal{F}\left(\bar{t}_{[\bar{l}, \bar{r}]}\right)= & \sum_{l_{N-1} \leq s_{N-1} \leq r_{N-1}} \ldots \sum_{l_{1} \leq s_{1} \leq r_{1}} \prod_{1 \leq a \leq N-1} \frac{\left(r_{a}-l_{a}\right) !}{\left(s_{a}-l_{a}\right) !\left(r_{a}-s_{a}\right) !} \\
& \times \overline{\operatorname{Sym}}_{\bar{t}_{[\bar{l}, \bar{r}]}}\left(Z_{\bar{s}}\left(\bar{t}_{[\bar{l}, \bar{r}]}\right) P_{f}^{-}\left(\mathcal{F}\left(\bar{t}_{[\bar{s}, \bar{r}]}\right)\right) \cdot P_{f}^{+}\left(\mathcal{F}\left(\bar{t}_{[\bar{l}, \bar{s}]}\right)\right)\right),
\end{aligned}
$$

where

$$
Z_{\bar{s}}\left(\bar{t}_{[\bar{l}, \bar{r}]}\right)=\prod_{a=1}^{N-2} \prod_{\substack{s_{a}<\ell \leq r_{a} \\ l_{a+1}<\ell^{\prime} \leq s_{a+1}}} \frac{q-q^{-1} t_{\ell}^{a} / t_{\ell^{\prime}}^{a+1}}{1-t_{\ell}^{a} / t_{\ell^{\prime}}^{a+1}}
$$

Equality (3.21) was proved in [12] and the proof is based on the current coproduct property (2.13) and the exchange relations between currents. 


\subsection{Special presentation of the universal weight function}

Let $\bar{m}=\left\{m_{1}, \ldots, m_{N-1}\right\}$ be a collection of the non-negative integers satisfying the admissibility condition

$$
m_{1} \geq m_{2} \geq m_{3} \ldots \geq m_{N-1} \geq m_{N}=0 .
$$

We define a series depending on the set of the variables $\bar{t}_{[\bar{m}]}$ of the form

$$
\tilde{X}\left(\bar{t}_{[\bar{m}]}\right)=\prod_{a=1}^{N-2} V\left(t_{m_{a+1}}^{a+1}, \ldots, t_{1}^{a+1} ; t_{m_{a}}^{a}, \ldots, t_{m_{a}-m_{a+1}+1}^{a}\right)
$$

where the rational series $V(\cdot ; \cdot)$ is given by the formulae

$$
\begin{aligned}
\tilde{V}\left(t_{k}^{2}, \ldots, t_{1}^{2} ; t_{k}^{1}, \ldots, t_{1}^{1}\right) & =\prod_{m=1}^{k}\left(\frac{1}{1-t_{m}^{1} / t_{m}^{2}} \prod_{m^{\prime}=m+1}^{k} \frac{q-q^{-1} t_{m^{\prime}}^{1} t_{m}^{2}}{1-t_{m^{\prime}}^{1} / t_{m}^{2}}\right) \\
& =\prod_{m=1}^{k}\left(\frac{1}{1-t_{m}^{1} / t_{m}^{2}} \prod_{m^{\prime}=1}^{m-1} \frac{q-q^{-1} t_{m}^{1} / t_{m^{\prime}}^{2}}{1-t_{m}^{1} / t_{m^{\prime}}^{2}}\right) .
\end{aligned}
$$

Define a normalized ordered product of the composed currents:

$$
\tilde{\mathcal{S}}_{\bar{m}}\left(\bar{t}_{[\bar{m}]}\right)=\tilde{X}\left(\bar{t}_{[\bar{m}]}\right) \prod_{N \geq a>1}\left(\frac{1}{\left(m_{a-1}-m_{a}\right) !} \prod_{m_{1}-m_{a} \geq \ell>m_{1}-m_{a-1}} F_{a, 1}\left(t_{\ell}^{1}\right)\right)
$$

This ordered product was called the dual string in the paper [13]. Denote the negative projections of the $q$-symmetrized dual strings (3.26) as follows

$$
\mathcal{E}_{m_{1}, m_{2}, \ldots, m_{N-1}}\left(\bar{t}_{[\bar{m}]}\right)=P_{f}^{-}\left(\overline{\operatorname{Sym}}_{\bar{t}_{[\bar{m}]}}\left(\tilde{\mathcal{S}}_{\bar{m}}\left(\bar{t}_{[\bar{m}]}\right)\right)\right) .
$$

Denote by $\mathcal{D}_{n_{1}, \ldots, n_{N-1}}\left(\bar{t}_{[\bar{n}]}\right)$ the elements of $U_{f}^{-}$defined by the recursive relations

$$
\begin{aligned}
& \overline{\operatorname{Sym}}_{\bar{t}_{[\bar{n}]}}\left(\sum_{\substack{n_{1} \geq m_{1} \geq 0 \\
m_{1} \geq \cdots \geq m_{N-1}}} \cdots \sum_{\substack{n_{N-1} \geq m_{N-1} \geq 0 \\
m_{1}}}\right. \\
& \left.Z_{\bar{m}}\left(\bar{t}_{[\bar{n}]}\right) \mathcal{D}_{n_{1}-m_{1}, \ldots, n_{N-1}-m_{N-1}}\left(\bar{t}_{[\bar{m}, \bar{n}]}\right) \cdot \mathcal{E}_{m_{1}, \ldots, m_{N-1}}\left(\bar{t}_{[\bar{m}]}\right)\right)=0 .
\end{aligned}
$$

It was proved in [13] that the coefficients $\mathcal{D}_{n_{1}, \ldots, n_{N-1}}\left(\bar{t}_{[\bar{n}]}\right)$ defined by $(3.28)$ are non-zero only iff $n_{1} \geq n_{2} \geq \cdots \geq n_{N-1} \geq 0$ and can be defined uniquely by means 
of (3.28) from the initial condition $\mathcal{D}_{0, \ldots, 0}\left(\bar{t}_{[\bar{n}]}\right)=1$. In particular,

$$
\begin{aligned}
\mathcal{D}_{m \text { times }}^{1, \ldots, 1,0, \ldots, 0}\left(t^{1}, \ldots, t^{N-1}\right) & =-\mathcal{E}_{\underbrace{1, \ldots, 1,0, \ldots, 0}_{m \text { times }}}\left(t^{1}, \ldots, t^{N-1}\right) \\
& =-\prod_{a=1}^{m-1} \frac{1}{1-t^{a} / t^{a+1}} P_{f}^{-}\left(F_{m+1,1}\left(t^{1}\right)\right) .
\end{aligned}
$$

Denote by $\bar{t}_{\left[\bar{s}^{\prime}\right]}$ the following collection of the formal variables

$$
\bar{t}_{\left[\bar{s}^{\prime}\right]}=\left\{t_{1}^{2}, \ldots, t_{s_{2}}^{2} ; \ldots ; t_{1}^{N-1}, \ldots, t_{s_{N-1}}^{N-1}\right\}
$$

excluding the variables of type 1 . We formulate without proof the following

Proposition 3.5 ([13]). There is a formal series identity

$$
\begin{aligned}
\mathcal{W}_{N}\left(\bar{t}_{[n]}\right)= & \sum_{\bar{s}} \prod_{a=1}^{N-1} \frac{n_{a} !}{s_{a} !} \overline{\operatorname{Sym}}_{\bar{t}_{[n]}}\left(Z_{\bar{s}}\left(\bar{t}_{\overline{[}}\right)\right. \\
& \left.\times \mathcal{D}_{n_{1}-s_{1}, \ldots, n_{N-1}-s_{N-1}}\left(\bar{t}_{[\bar{s}, \bar{n}]}\right) \cdot \mathcal{W}_{N-1}\left(\bar{t}_{\left[s^{\prime}\right]}\right) \cdot F_{1}\left(t_{s_{1}}^{1}\right) \cdots F_{1}\left(t_{1}^{1}\right)\right) .
\end{aligned}
$$

In this paper we will need the following corollary of this proposition. Let $\bar{t}_{\left[\bar{n}^{\prime}\right]_{m}}$ be the following collection of formal variables

$$
\begin{aligned}
& \bar{t}_{\left[\bar{n}^{\prime}\right]_{m}}=\left\{t_{1}^{2}, \ldots, t_{n_{2}-1}^{2} ; \ldots ;\right. \\
&\left.t_{1}^{m}, \ldots, t_{n_{m}-1}^{m} ; t_{1}^{m+1}, \ldots, t_{n_{m+1}}^{m+1} ; \ldots ; t_{1}^{N-1}, \ldots, t_{n_{N-1}}^{N-1}\right\} .
\end{aligned}
$$

Note that $\bar{t}_{\left[\bar{n}^{\prime}\right]_{1}} \equiv \bar{t}_{\left[\bar{n}^{\prime}\right]}$.

\section{Corollary 3.6.}

$$
\begin{aligned}
P_{f}^{+}\left(\mathcal{F}\left(\bar{t}_{[\bar{n}]}\right)\right)= & P_{f}^{+}\left(\mathcal{F}\left(\bar{t}_{\left[\bar{n}^{\prime}\right]_{1}}\right)\right) \cdot \mathcal{F}\left(\bar{t}_{\left[n_{1}\right]}^{1}\right)-\sum_{m=1}^{N-1} \prod_{a=1}^{m}\left(n_{a}\right) \overline{\operatorname{Sym}}_{\bar{t}_{[\bar{n}]}}\left(P_{f}^{-}\left(F_{m+1,1}\left(t_{n_{1}}^{1}\right)\right)\right. \\
& \left.\times P_{f}^{+}\left(\mathcal{F}\left(\bar{t}_{\left[\bar{n}^{\prime}\right]_{m}}\right)\right) \cdot \mathcal{F}\left(\bar{t}_{\left[n_{1}-1\right]}^{1}\right) \cdot \mathbb{Z}_{m}\left(\bar{t}_{[\bar{n}]}\right)\right)+\mathbb{W},
\end{aligned}
$$

where

$$
\mathbb{Z}_{m}\left(\bar{t}_{[\bar{n}]}\right)=\prod_{a=1}^{m-1}\left(\frac{1}{1-t_{n_{a}}^{a} / t_{n_{a+1}}^{a+1}} \prod_{j=1}^{n_{a+1}-1} \frac{q-q^{-1} t_{n_{a}}^{a} / t_{j}^{a+1}}{1-t_{n_{a}}^{a} / t_{j}^{a+1}}\right) \prod_{j=1}^{n_{m+1}} \frac{q-q^{-1} t_{n_{m}}^{m} / t_{j}^{m+1}}{1-t_{n_{m}}^{m} / t_{j}^{m+1}}
$$

and the terms $\mathbb{W}$ in $(3.32)$ are such that $P_{f}^{+}\left(: \mathcal{T}_{N}(t) \cdot \mathbb{W}:\right)=0$.

Recall that $\mathcal{F}\left(\vec{t}_{\left[n_{1}\right]}^{1}\right)=F_{1}\left(t_{n_{1}}^{1}\right) \cdots F_{1}\left(t_{1}^{1}\right)$ and $\mathcal{F}\left(\vec{t}_{\left[n_{1}-1\right]}^{1}\right)=F_{1}\left(t_{n_{1}-1}^{1}\right) \cdots F_{1}\left(t_{1}^{1}\right)$.

The first term in the right hand side of (3.32) corresponds to the term with all $s_{a}=n_{a}$ in (3.30). Each of the terms in the summation over $m$ in (3.32) corresponds to the following values of $s_{m}$ in the general formula (3.30): $s_{1}=$ $n_{1}-1, \ldots, s_{m}=n_{m}-1$ and $s_{m+1}=n_{m+1}, \ldots, s_{N-1}=n_{N-1}$. The corresponding elements $\mathcal{D}_{1, \ldots, 1,0, \ldots, 0}$ are given by (3.29), which brings in (3.32) the product of the rational factors $\left(1-t_{n_{a}}^{a} / t_{n_{a+1}}^{a+1}\right)^{-1}$. Other rational factors are given by the series $Z_{\bar{s}}\left(\bar{t}_{[\bar{n}]}\right)$ for these particular values of $\bar{s}$. 
The general structure of the terms $\mathbb{W}$ which are not presented explicitly in the right hand side of (3.32) can be described as follows. The structure of the coefficients $\mathcal{E}_{m_{1}, m_{2}, \ldots, m_{N-1}}\left(\bar{t}_{[\bar{m}]}\right)$ (3.27) implies that these terms will have on the left the negative projections of the string $\tilde{\mathcal{S}}_{\bar{m}}\left(\bar{t}_{[\bar{m}]}\right)$ which contains, at least, the product of two currents $F_{c_{1}, 1}$ and $F_{c_{2}, 1}$ or the product of the several negative projections of the strings of type (3.27). Since the projection of the string can be always factorized to the product of the projection of the currents [12], the general structure of the terms $\mathbb{W}$ will be $\mathbb{W}=\sum P_{f}^{-}\left(F_{c_{1}, 1}\right) \cdot P_{f}^{-}\left(F_{c_{2}, 1}\right) \cdot \mathbb{W}^{\prime}$. The elements $\mathbb{W}^{\prime}$ are some elements of $U_{F}$ and their exact structure is unimportant. The reason why $P_{f}^{+}\left(: \mathcal{T}_{N}(t) \cdot \mathbb{W}:\right)=0$ will be explained in the next subsection.

Note that the identity (3.30) can be proved directly using only the ordering relations (3.21) and the rules of calculations of the negative projections from the product of currents. Indeed, for an arbitrary product of currents $\mathcal{F}\left(\bar{t}_{[\bar{n}]}\right)$, these ordering relations can be written in the form

$$
P_{f}^{+}\left(\mathcal{F}\left(\bar{t}_{[\bar{n}]}\right)\right)=\mathcal{F}\left(\bar{t}_{[\bar{n}]}\right)-\sum P_{f}^{-}\left(\mathcal{F}^{\prime}\right) \cdot P_{f}^{+}\left(\mathcal{F}^{\prime \prime}\right),
$$

where the number of currents in the product $\mathcal{F}^{\prime \prime}$ is less than in the original product $\mathcal{F}\left(\bar{t}_{[\bar{n}]}\right)$. Thus, one can replace recursively the positive projection $P_{f}^{+}\left(\mathcal{F}^{\prime \prime}\right)$ by the right hand side of the relation (3.34) up to the obvious identity $P_{f}^{+}\left(F_{i}(t)\right)=$ $F_{i}(t)-P_{f}^{-}\left(F_{i}(t)\right)$ valid for arbitrary simple current $F_{i}(t)$. Calculating the negative projections $P_{f}^{-}\left(\mathcal{F}^{\prime}\right)$ to obtain the projections of the strings of type (3.27), we can prove the identity (3.30) by brute force calculations. The technique of the generating series developed in [13] yields more elegant way of proving this and many other similar identities.

Example 3.7. Let us present an example of the general formula (3.30) in the case $N=3, n_{1}=2$ and $n_{2}=2$. To reduce the formula we will use shorthand notations $P_{f}^{ \pm}(\cdot)=[\cdot]^{ \pm}$. We also denote $t_{i}^{2}=s_{i}$ and $t_{i}^{1}=t_{i}$ and $\overline{\text { Sym }}$ below will be the $q$-symmetrization over variables $t_{i}$ and $s_{i}$.

$$
\begin{gathered}
{\left[F_{2}\left(s_{2}\right) F_{2}\left(s_{1}\right) F_{1}\left(t_{2}\right) F_{1}\left(t_{1}\right)\right]^{+}=\left[F_{2}\left(s_{2}\right) F_{2}\left(s_{1}\right)\right]^{+} F_{1}\left(t_{2}\right) F_{1}\left(t_{1}\right)} \\
-2 \overline{\operatorname{Sym}}\left(\left[F_{1}\left(t_{2}\right)\right]^{-}\left[F_{2}\left(s_{2}\right) F_{2}\left(s_{1}\right)\right]^{+} F_{1}\left(t_{1}\right) \prod_{j=1}^{2} \frac{q^{-1} t_{2}-q s_{j}}{t_{2}-s_{j}}\right) \\
-4 \overline{\operatorname{Sym}}\left(\left[F_{3,1}\left(t_{2}\right)\right]^{-}\left[F_{2}\left(s_{1}\right)\right]^{+} F_{1}\left(t_{1}\right) \frac{s_{2}}{s_{2}-t_{2}} \frac{q^{-1} t_{2}-q s_{1}}{t_{2}-s_{1}}\right) \\
+\left\{\overline { \operatorname { S y m } } \left(\left(\left[F_{1}\left(t_{2}\right)\right]^{-}\left[F_{1}\left(t_{1}\right)\right]^{-}-\frac{1}{2}\left[F_{1}\left(t_{2}\right) F_{1}\left(t_{1}\right)\right]^{-}\right)\right.\right. \\
\left.\times\left[F_{2}\left(s_{2}\right) F_{2}\left(s_{1}\right)\right]^{+} \prod_{i, j=1}^{2} \frac{q^{-1} t_{i}-q s_{j}}{t_{i}-s_{j}}\right)
\end{gathered}
$$




$$
\begin{aligned}
& +4 \overline{\operatorname{Sym}}\left(\left(\frac{s_{2}}{s_{2}-t_{2}}\left[F_{3,1}\left(t_{2}\right)\right]^{-}\left[F_{1}\left(t_{1}\right)\right]^{-}+\frac{s_{2}}{s_{2}-t_{1}} \frac{q^{-1} t_{2}-q s_{2}}{t_{2}-s_{2}}\left[F_{1}\left(t_{2}\right)\right]^{-}\right.\right. \\
& \left.\left.\quad \times\left[F_{3,1}\left(t_{1}\right)\right]^{-}-\frac{s_{2}}{s_{2}-t_{2}}\left[F_{3,1}\left(t_{2}\right) F_{1}\left(t_{1}\right)\right]^{-}\right)\left[F_{2}\left(s_{1}\right)\right]^{+} \prod_{i=1}^{2} \frac{q^{-1} t_{i}-q s_{1}}{t_{i}-s_{1}}\right) \\
& +4 \overline{\operatorname{Sym}}\left(\left(\left[F_{3,1}\left(t_{2}\right)\right]^{-}\left[F_{3,1}\left(t_{1}\right)\right]^{-}-\frac{1}{2}\left[F_{3,1}\left(t_{2}\right) F_{3,1}\left(t_{1}\right)\right]^{-}\right)\right. \\
& \left.\left.\quad \times \frac{s_{1}}{s_{1}-t_{1}} \frac{s_{2}}{s_{2}-t_{2}} \frac{q^{-1} t_{2}-q s_{1}}{t_{2}-s_{1}}\right)\right\} .
\end{aligned}
$$

The terms in curly brackets correspond to the term $\mathbb{W}$ in (3.32).

3.4. The action of $\mathrm{L}_{a, b}^{+}(t)$ onto $P_{f}^{-}\left(F_{c, d}(t)\right)$

Definition 3.8. Let $I$ be the right ideal of $\bar{U}_{q}\left(\widehat{\mathfrak{g l}}_{N}\right)$, generated by all elements of the form $F_{i}[n] \cdot U_{q}\left(\mathfrak{b}^{+}\right)$such that $i=1, \ldots, N-1$ and $n<0$. We denote equalities modulo elements from the ideal $I$ by the symbol ' $\sim_{I}$ '.

Proposition 3.9. We have an equivalence

$$
\mathrm{L}_{a, b}^{+}(t) \cdot \mathrm{F}_{c, d}^{-}(s) \sim_{I} \delta_{a, c} \frac{\left(q-q^{-1}\right) s}{s-t} \mathrm{~L}_{d, b}^{+}(t) .
$$

One of our technical tools will be the rule of commuting the negative projections of the composed currents $P_{f}^{-}\left(F_{c, d}\left(t^{\prime}\right)\right)$ with matrix elements of the fundamental L-operator $\mathrm{L}_{a, b}^{+}(t)$. We need a result of this calculation only modulo elements from the ideal $I$ and call this as action of $P_{f}^{-}\left(F_{c, d}\left(t^{\prime}\right)\right)$ onto $\mathrm{L}_{a, b}^{+}(t)$. Due to the relation (3.6), in order to calculate the action of the matrix elements $\mathrm{L}_{a, b}^{+}(t)$ onto $P_{f}^{-}\left(F_{c, d}\left(t^{\prime}\right)\right)$ one has to calculate first the action of the matrix elements $\mathrm{L}_{a, b}^{+}(t)$ onto Gauss coordinates $\mathrm{F}_{c, d}^{-}\left(t^{\prime}\right)$.

Proof of Proposition 3.9 will be done considering each fixed $a$.

Fix $a$ and consider $c<a$. This case is simple. Formulas (3.6) can be inverted to express the Gauss coordinates $\mathrm{F}_{c, d}^{-}(s)$ in terms of the modes of the currents $F_{d}\left[n_{d}\right], \ldots, F_{c-1}\left[n_{c-1}\right]$. But the L-operator modes $\mathrm{L}_{a, b}^{+}[n]$ simply commute with these current modes and so $\mathrm{L}_{a, b}^{+}(t) \cdot \mathrm{F}_{c, d}^{-}(s)=\mathrm{F}_{c, d}^{-}(s) \cdot \mathrm{L}_{a, b}^{+}(t) \in I$.

The case when $a=N$ is also simple. For this choice, we have also $b=N$ and $\mathrm{L}_{N, N}^{+}(t) \equiv k_{N}^{+}(t)$ commutes with the Gauss coordinates $\mathrm{F}_{c, d}^{-}(s)$ for $c=2, \ldots, N-1$. It means that $\mathrm{L}_{N, N}^{+}(t) \cdot \mathrm{F}_{c, d}^{-}(s) \sim 0$ for $c<N$. Let $c=N$. Taking into account that $\mathrm{F}_{N, d}^{-}(s)=\mathrm{L}_{d, N}^{-}(s) \mathrm{L}_{N, N}^{-}(s)^{-1}$ and the commutation relation

$$
\mathrm{L}_{N, N}^{+}(t) \mathrm{L}_{d, N}^{-}(s)=\frac{q t-q^{-1} s}{t-s} \mathrm{~L}_{d, N}^{-}(s) \mathrm{L}_{N, N}^{+}(t)-\frac{\left(q-q^{-1}\right) s}{t-s} \mathrm{~L}_{d, N}^{+}(t) \mathrm{L}_{N, N}^{-}(s)
$$

we prove the statement of the proposition for $a=N$. 
Let $a<N$ and $c>a$. First consider the case when $b>c>a$. We have

$$
\mathrm{L}_{a, b}^{+}(t) \cdot \mathrm{F}_{c, d}^{-}(s)=\mathrm{F}_{b, a}^{+}(t) \mathrm{F}_{c, d}^{-}(s) k_{b}^{+}(t)+\sum_{j=b+1} \mathrm{~F}_{j, a}^{+}(t) \mathrm{F}_{c, d}^{-}(s) k_{j}^{+}(t) E_{b, j}^{+}(t)
$$

due to the commutativity of the Gauss coordinates $k_{b}^{+}(t)$ and $k_{j}^{+}(t) E_{b, j}^{+}(t)$ with modes of the currents $F_{d}\left[n_{d}\right], \ldots, F_{c-1}\left[n_{c-1}\right]$ or with Gauss coordinates $\mathrm{F}_{c, d}^{-}(s)$. The statement of the proposition follows from the lemma.

Lemma 3.10. For $b>c>d>a$ and $b>c>a \geq d$

$$
\mathrm{F}_{b, a}^{+}(t) \mathrm{F}_{c, d}^{-}(s) \sim_{I} F_{b, a}(t) \mathrm{F}_{c, d}^{-}(s) \in I .
$$

Proof is based on the commutation relations of the composed currents $F_{b, a}(t)$ and $F_{c, d}(s)$. They are

$$
\begin{gathered}
\frac{t-s}{q t-q^{-1} s} F_{b, a}(t) F_{c, d}(s)=\frac{q^{-1} t-q s}{t-s} F_{c, d}(s) F_{b, a}(t), \quad d<a, \\
F_{b, a}(t) F_{c, d}(s)=\frac{q^{-1} t-q s}{t-s} F_{c, d}(s) F_{b, a}(t), \quad d=a, \\
F_{b, a}(t) F_{c, d}(s)=F_{c, d}(s) F_{b, a}(t), \quad d>a,
\end{gathered}
$$

and they take into account the Serre relations (2.12) (see details in Appendix A of the paper [12]). The product $F_{b, a}(t) F_{c, d}(s)$ has no pole for $d \geq a$ and has first order pole at the point $t=s$ in the case $d<a$, but the residue at this point of this product is zero. It means that commuting negative projections of the current $P_{f}^{-}\left(F_{c, d}(s)\right)$ through the total currents $F_{b, a}(t)$ no higher currents will be created and the result of commutation will belong to the right ideal $I$. Because of the relation between negative Gauss coordinates and the negative projections of the composed currents given by (3.6) the same statement will be true for the Gauss coordinates.

Next we consider the cases when $c>a$ and $c \geq b$. The statement of Proposition 3.9 will be proved by a double induction over $c$ starting from $c=N$ and over $b$ starting from $c$. Let $c=N$ and $b=N$. Then using again the fact $\mathrm{F}_{N, d}^{-}(s)=\mathrm{L}_{d, N}^{-}(s) k_{N}^{-}(s)^{-1}$ and the commutation relations

$$
\mathrm{L}_{a, N}^{+}(t) \mathrm{L}_{d, N}^{-}(s)=\frac{t-s}{q^{-1} t-q s} \mathrm{~L}_{d, N}^{-}(s) \mathrm{L}_{a, N}^{+}(t)+\frac{\left(q-q^{-1}\right) s}{q^{-1} t-q s} \mathrm{~L}_{a, N}^{-}(t) \mathrm{L}_{d, N}^{+}(t)
$$

we obtain the inclusion $\mathrm{L}_{a, N}^{+}(t) \mathrm{F}_{N, d}^{-}(s) \in I$. Before considering other cases we prove the following lemma.

Lemma 3.11. For $b<c$ and arbitrary $a<b$ and $d<c$ we have

$$
\begin{array}{r}
\mathrm{L}_{a, b}^{+}(t) \cdot \mathrm{L}_{d, c}^{-}(s)+\frac{\left(q-q^{-1}\right)^{2} t s}{(t-s)^{2}} \\
\mathrm{~L}_{d, c}^{+}(t) \cdot \mathrm{L}_{a, b}^{-}(s) \sim_{I} 0, \quad a \neq d, \\
\mathrm{~L}_{a, b}^{+}(t) \cdot \mathrm{L}_{a, c}^{-}(s) \sim_{I} 0, \quad a=d .
\end{array}
$$


Proof. The case of $a=d$ follows from the relation

$$
\mathrm{L}_{a, b}^{+}(t) \mathrm{L}_{a, c}^{-}(s)=\frac{t-s}{q t-q^{-1} s} \mathrm{~L}_{a, c}^{-}(s) \mathrm{L}_{a, b}^{+}(t)+\frac{\left(q-q^{-1}\right) s}{q t-q^{-1} s} \mathrm{~L}_{a, b}^{-}(s) \mathrm{L}_{a, c}^{+}(t) .
$$

The case $a<d$ follows from two relations

$$
\begin{aligned}
& {\left[\mathrm{L}_{a, b}^{+}(t), \mathrm{L}_{d, c}^{-}(s)\right]=\frac{\left(q-q^{-1}\right)}{t-s}\left(s \mathrm{~L}_{a, c}^{+}(t) \mathrm{L}_{d, b}^{-}(s)-t \mathrm{~L}_{a, c}^{-}(s) \mathrm{L}_{d, b}^{+}(t)\right),} \\
& {\left[\mathrm{L}_{a, c}^{+}(t), \mathrm{L}_{d, b}^{-}(s)\right]=\frac{\left(q-q^{-1}\right) t}{t-s}\left(\mathrm{~L}_{d, c}^{-}(s) \mathrm{L}_{a, b}^{+}(s)-\mathrm{L}_{d, c}^{+}(t) \mathrm{L}_{a, b}^{-}(s)\right) .}
\end{aligned}
$$

The case $a>d$ can be proved analogously.

Return to the proof of Proposition 3.9. Keep $c=N$ and consider $b=N-1$. Then

$$
\begin{aligned}
& \mathrm{L}_{a, N-1}^{+}(t) \mathrm{F}_{N, d}^{-}(s)= \mathrm{L}_{a, N-1}^{+} \mathrm{L}_{d, N}^{-}(s) k_{N}^{-}(s)^{-1} \\
& \sim_{I}-\frac{\left(q-q^{-1}\right) t s}{(t-s)^{2}} \mathrm{~L}_{d, N}^{+}(t) \mathrm{L}_{a, N-1}^{-}(s) k_{N}^{-}(s)^{-1} \\
&=-\frac{\left(q-q^{-1}\right) t s}{(t-s)^{2}} \mathrm{~L}_{d, N}^{+}(t)\left(\mathrm{F}_{N-1, a}^{-}(s) k_{N-1}^{-}(s)\right. \\
&\left.+\mathrm{F}_{N, a}^{-}(s) k_{N}^{-}(s) \mathrm{E}_{N-1, N}^{-}(s)\right) k_{N}^{-}(s)^{-1},
\end{aligned}
$$

where we used Lemma 3.11. Now the first term in the right hand side of (3.38) belongs to the ideal $I$ because the second index of $\mathrm{L}_{d, N}^{+}(t)$ is bigger than the first index of $\mathrm{F}_{N-1, a}^{-}(s)$. The second term corresponds to the case $c=b=N$ considered above, and thus also belongs to $I$. Reducing $b$ and using the Lemma 3.11 we proved the statement for all $b<N=c$.

Let now $c=N-1$. For the negative Gauss coordinate $\mathrm{F}_{N-1, d}^{-}(s)$ we can use the formula

$$
\mathrm{F}_{N-1, d}^{-}(s)=\left(\mathrm{L}_{d, N-1}^{-}(s)-\mathrm{L}_{d, N}^{-}(s) \mathrm{E}_{N-1, N}^{-}(s)\right) k_{N-1}^{-}(s)^{-1} .
$$

To prove that the product $\mathrm{L}_{a, N-1}^{+}(t) \mathrm{L}_{d, N-1}^{-}(s) \in I$ we can use the same arguments as for the product $\mathrm{L}_{a, N}^{+}(t) \mathrm{L}_{d, N}^{-}(s)$. The fact that $\mathrm{L}_{a, N-1}^{+}(t) \mathrm{L}_{d, N}^{-}(s) \in I$ was already proved above. Continuing we check that $\mathrm{L}_{a, b}^{+}(t) \mathrm{L}_{d, N-1}^{-}(s) \in I$ for all $b<N-1$. For general $c$ we have to use instead of the formulae (3.39) the relation

$$
\mathrm{F}_{c, d}^{-}(s)=\mathrm{L}_{d, c}^{-}(s) k_{c}^{-}(s)^{-1}+\mathrm{L}_{d, c+1}^{-}(s) X_{c+1}+\cdots+\mathrm{L}_{d, N}^{-}(s) X_{N},
$$

where the explicit form of the elements $X_{j} \in U_{q}\left(\mathfrak{b}^{-}\right)$is not important.

At last we have to check the case $a=c<N$. The case $a=c=N$ was considered above. According to (3.40) the consideration of $\mathrm{L}_{a, b}^{+}(t) \mathrm{F}_{a, d}^{-}(s)$ reduces to the analysis of the product $\mathrm{L}_{a, b}^{+}(t) \mathrm{L}_{d, a}^{-}(s) k_{a}^{-}(s)^{-1}$. We have

$$
\left[\mathrm{L}_{a, b}^{+}(t), \mathrm{L}_{d, a}^{-}(s)\right]=\frac{\left(q-q^{-1}\right)}{t-s}\left(t \mathrm{~L}_{d, b}^{-}(s) \mathrm{L}_{a, a}^{+}(t)-s \mathrm{~L}_{d, b}^{+}(t) \mathrm{L}_{a, a}^{+}(t)\right) .
$$


Since $\mathrm{L}_{a, a}^{-}(s) k_{a}^{-}(s)^{-1}=1+\sum_{j=a+1}^{N} \mathrm{~F}_{j, a}^{-}(s) k_{j}^{-}(s) \mathrm{E}_{a, j}^{-}(s) k_{a}^{-}(s)^{-1}$ and $\mathrm{L}_{d, b}^{+}(t) \mathrm{F}_{j, a}^{-}(s)$ $\in I$ the statement of the Proposition 3.9 is proved.

Corollary 3.12. We have an equivalence

$$
\mathrm{L}_{a, b}^{+}(t) \cdot P_{f}^{-}\left(F_{c, d}(s)\right) \sim_{I} \delta_{a, c} \frac{\left(q-q^{-1}\right)^{c-d-1} s}{t-s} \mathrm{~L}_{d, b}^{+}(t) .
$$

Proof. Let us apply the matrix element $\mathrm{L}_{a, b}^{+}(t)$ to both side of (3.6). The first term gives the right hand side of (3.41). Other terms produce products of the Kronecker's symbols $\delta_{a, i_{1}} \delta_{d, i_{2}} \delta_{i_{1}, i_{3}} \cdots \delta_{i_{\ell-1}, c}$ which are zero due to the restriction $d<i_{1}<\cdots<i_{\ell}<c$. If $\ell=1$ then $\delta_{a, i_{1}} \delta_{d, c}=0$ since $d<c$.

Let us explain why $P_{f}^{+}\left(: \mathcal{T}_{N}(t) \cdot \mathbb{W}:\right)=0$, where $\mathbb{W}$ are the terms not shown explicitly in the right hand side of (3.32). Due to Corollary 3.12 the action of $\mathrm{L}_{a, b}^{+}(t)$ onto the product of two negative projections of the currents $P_{f}^{-}\left(F_{c_{1}, 1}\right) \cdot P_{f}^{-}\left(F_{c_{2}, 1}\right)$ is proportional to the product of delta-symbols: $\delta_{a, c_{1}} \delta_{1, c_{2}}$. But since $c_{2}>1$ this is zero modulo elements of the right ideal $I$, which obviously satisfies $P_{f}^{+}(I)=0$.

\section{Ordering of the universal objects}

The proof of main Theorem 1 consists of a detailed analysis of the circular ordering of the product of the transfer matrix and of the universal Bethe vectors expressed in terms of the current generators of the quantum affine algebra $\bar{U}_{q}\left(\widehat{\mathfrak{g l}}_{N}\right)$. In the next two subsections we will perform such an analysis to the case $N=2$ and prove main Theorem 1 for the algebra $\bar{U}_{q}\left(\widehat{\mathfrak{g l}}_{2}\right)$. Then we go on by induction over $N$.

In what follows, besides the right ideal $I$ and the left ideal $J$ introduced in Definition 3.8 and Definition 2.2, we will also use the following ideal $K$.

Definition 4.1. We denote by $K$ the two-sided $\bar{U}_{q}\left(\widehat{\mathfrak{g l}}_{N}\right)$ ideal generated by the elements which have at least one arbitrary mode $k_{i}^{-}[n], i=1, \ldots, N, n \leq 0$, of the negative Cartan current $k_{i}^{-}(t)$.

Equalities in $\bar{U}_{q}\left(\widehat{\mathfrak{g l}}_{N}\right)$ modulo element of the ideal $K$ are denoted by the symbol ' $\sim_{K}$ '.

Equalities in $\bar{U}_{q}\left(\widehat{\mathfrak{g l}}_{N}\right)$ modulo the right ideal $I$, the left ideal $J$ and the twosided ideal $K$ will be denoted by the symbol ' $\approx$ '.

4.1. Ordering for $\bar{U}_{q}\left(\widehat{\mathfrak{g l}}_{2}\right)$

The algebra $\bar{U}_{q}\left(\widehat{\mathfrak{g l}}_{2}\right)$ is generated by the modes of the Gauss coordinates $k_{1}^{ \pm}(t)$, $k_{2}^{ \pm}(t), \mathrm{E}_{12}^{ \pm}(t), \mathrm{F}_{21}^{ \pm}(t)$ in the L-operator realization or by the modes of the currents $k_{1}^{ \pm}(t), k_{2}^{ \pm}(t), E_{1}(t), F_{1}(t)$ in the current realization. The standard quantum affine algebra $U_{q}\left(\widehat{\mathfrak{g l}}_{2}\right)$ can be obtained from $\bar{U}_{q}\left(\widehat{\mathfrak{g l}}_{2}\right)$ by imposing the restriction $k_{i}^{+}[0] k_{i}^{-}[0]=1, i=1,2$. To simplify further formulas we shall not use index of the single simple root in the notation of the Gauss coordinates and the currents, that is 
we use the following identification: $\mathrm{E}_{12}^{ \pm}(t) \equiv \mathrm{E}^{ \pm}(t), \mathrm{F}_{21}^{ \pm}(t) \equiv \mathrm{F}^{ \pm}(t), E_{1}(t) \equiv E(t)$, $F_{1}(t) \equiv F(t)$. Let $\psi^{ \pm}(t)=k_{1}^{+}(t) k_{2}^{+}(t)^{-1}$.

The universal transfer matrix for $\bar{U}_{q}\left(\widehat{\mathfrak{g l}}_{2}\right)$ is given by the relation

$\mathcal{T}_{2}(t)=\mathrm{L}_{11}^{+}(t)+\mathrm{L}_{22}^{+}(t) \quad$ with $\quad \mathrm{L}_{11}^{+}=k_{1}^{+}(t)+\mathrm{F}^{+}(t) k_{2}^{+}(t) \mathrm{E}^{+}(t), \quad \mathrm{L}_{22}^{+}(t)=k_{2}^{+}(t)$, while a universal weight function is a projection $P_{f}^{+}(\mathcal{F}(\bar{t}))=P_{f}^{+}\left(F\left(t_{n}\right) \cdots F\left(t_{1}\right)\right)$. We avoid to use upper index in the notation of the formal variables $t_{j}$.

Proposition 4.2. There is a formal series equality in the algebra $\bar{U}_{q}\left(\widehat{\mathfrak{g l}}_{2}\right)$

$$
\begin{aligned}
\mathcal{T}_{2}(t) \cdot P_{f}^{+}(\mathcal{F}(\bar{t})) \approx & P_{f}^{+}(\mathcal{F}(\bar{t}))\left(\prod_{i=1}^{n} \frac{q^{-1}-q t_{i} / t}{1-t_{i} / t} k_{1}^{+}(t)+\prod_{i=1}^{n} \frac{q-q^{-1} t_{i} / t}{1-t_{i} / t} k_{2}^{+}(t)\right) \\
& +n \overline{\operatorname{Sym}}_{\bar{t}}\left(\mathrm{~F}^{+}(t) k_{2}^{+}(t) F\left(t_{n}\right) \cdots F\left(t_{2}\right) \frac{\left(q-q^{-1}\right) t_{1} / t}{1-t_{1} / t} \psi^{+}\left(t_{1}\right)\right) \\
& -n \overline{\operatorname{Sym}}_{\bar{t}}\left(\mathrm{~F}^{+}(t) k_{2}^{+}(t) F\left(t_{n-1}\right) \cdots F\left(t_{1}\right) \frac{\left(q-q^{-1}\right) t_{n} / t}{1-t_{n} / t}\right) \cdot(4.1)
\end{aligned}
$$

Proof of Proposition 4.2 is based on the special presentation of the universal weight function given by the Corollary 3.6. In this case we have

$$
P_{f}^{+}(\mathcal{F}(\bar{t}))=F\left(t_{n}\right) \cdots F\left(t_{1}\right)-n \overline{\operatorname{Sym}}_{\bar{t}}\left(P_{f}^{-}\left(F\left(t_{n}\right)\right) \cdot F\left(t_{n-1}\right) \cdots F\left(t_{1}\right)\right)+\mathbb{W},
$$

where $\mathbb{W}$ are the terms which have on the left the product of at least two negative projections of the currents $F(t)$. Since $P_{f}^{-}(F(t))=-\mathrm{F}^{-}(t)$ these terms can be equally described as having on the left the product of at least of two negative Gauss coordinates $\mathrm{F}^{-}(t)$. As was explained above and as we will see explicitly below, the terms $\mathbb{W}$ are characterized by the property that $\mathcal{T}_{2}(t) \cdot \mathbb{F} \in I$.

We will order the product of $\mathcal{T}_{2}(t)$ and each summand in the right hand side of (4.2) separately. For the ordering of the first term we use the relation

$$
\left[\mathrm{E}^{+}(t), F\left(t_{1}\right)\right]=\frac{\left(q-q^{-1}\right) t_{1}}{t-t_{1}}\left(\psi^{+}\left(t_{1}\right)-\psi^{-}\left(t_{1}\right)\right),
$$

so that

$$
\begin{aligned}
{\left[k_{1}^{+}(t)\right.} & \left.+k_{2}^{+}(t)+\mathrm{F}^{+}(t) k_{2}^{+}(t) \mathrm{E}^{+}(t)\right] \cdot F\left(t_{n}\right) \cdots F\left(t_{1}\right) \\
= & F\left(t_{n}\right) \cdots F\left(t_{1}\right)\left(\prod_{i=1}^{n} \frac{q^{-1} t-q t_{i}}{t-t_{i}} k_{1}^{+}(t)+\prod_{i=1}^{n} \frac{q t-q^{-1} t_{i}}{t-t_{i}} k_{2}^{+}(t)\right) \\
& +n \overline{\operatorname{Sym}}_{\bar{t}}\left(\mathrm{~F}^{+}(t) k_{2}^{+}(t) F\left(t_{n}\right) \cdots F\left(t_{2}\right) \frac{\left(q-q^{-1}\right) t_{1}}{t-t_{1}} \psi^{+}\left(t_{1}\right)\right) \\
& -n \overline{\operatorname{Sym}}_{\bar{t}}\left(\mathrm{~F}^{+}(t) k_{2}^{+}(t) F\left(t_{n}\right) \cdots F\left(t_{2}\right) \frac{\left(q-q^{-1}\right) t_{1}}{t-t_{1}} \psi^{-}\left(t_{1}\right)\right) \\
& +\mathrm{F}^{+}(t) k_{2}^{+}(t) F\left(t_{n}\right) \cdots F\left(t_{1}\right) \mathrm{E}^{+}(t) .
\end{aligned}
$$


Here we used the exchange relations of the Cartan currents $k_{i}^{+}(t)$ and the total currents $F\left(t_{j}\right)$ and $q$-symmetrization in second and third terms of the right hand side of (4.3) appears when commuting Cartan currents $\psi^{ \pm}\left(t_{i}\right)$ with total currents $F\left(t_{j}\right)$.

Observe that the last term in (4.3) belongs to the ideal $J$ and the next to last term belongs to the ideal $K$. At this point we benefit from the absence of the restriction (2.14) in the algebra $\bar{U}_{q}\left(\widehat{\mathfrak{g l}}_{2}\right)$. Otherwise we would have to take into account the zero modes of the currents $\psi^{-}\left(t_{1}\right)$ if we were considering the standard quantum affine algebra $U_{q}\left(\widehat{\mathfrak{g l}}_{2}\right)$.

According to (3.21)

$$
F\left(t_{n}\right) \cdots F\left(t_{1}\right) \sim_{I} P_{f}^{+}\left(F\left(t_{n}\right) \cdots F\left(t_{1}\right)\right)
$$

and equality (4.3) implies the equivalence

$$
\begin{aligned}
& {\left[k_{1}^{+}(t)+k_{2}^{+}(t)+\mathrm{F}^{+}(t) k_{2}^{+}(t) \mathrm{E}^{+}(t)\right] \cdot F\left(t_{n}\right) \cdots F\left(t_{1}\right)} \\
& \approx P_{f}^{+}\left(F\left(t_{n}\right) \cdots F\left(t_{1}\right)\right)\left(\prod_{i=1}^{n} \frac{q^{-1}-q t_{i} / t}{1-t_{i} / t} k_{1}^{+}(t)+\prod_{i=1}^{n} \frac{q-q^{-1} t_{i} / t}{1-t_{i} / t} k_{2}^{+}(t)\right) \\
& \quad+n \overline{\operatorname{Sym}}_{\bar{t}}\left(\mathrm{~F}^{+}(t) k_{2}^{+}(t) F\left(t_{n}\right) \cdots F\left(t_{2}\right) \frac{\left(q-q^{-1}\right) t_{1} / t}{1-t_{1} / t} \psi^{+}\left(t_{1}\right)\right)
\end{aligned}
$$

Consider now the ordering of the product $\mathcal{T}_{2}(t)\left(P_{f}^{-}\left(F\left(t_{n}\right)\right) \cdot F\left(t_{n-1}\right) \cdots F\left(t_{1}\right)\right)$. To perform this we will use the following specialization of the Proposition 3.9. For arbitrary element $\mathbb{X} \in U_{F}$ and $m \geq 1$ we have

$$
\begin{aligned}
& \mathrm{L}_{11}^{+}(t) \cdot P_{f}^{-}\left(F\left(t_{m}\right) \cdots F\left(t_{1}\right)\right) \cdot \mathbb{X} \sim_{I} 0 \\
& \mathrm{~L}_{22}^{+}(t) \cdot P_{f}^{-}\left(F\left(t_{m}\right) \cdots F\left(t_{1}\right)\right) \cdot \mathbb{X} \sim_{I} \delta_{m 1} \frac{\left(q-q^{-1}\right) t_{1} / t}{1-t_{1} / t} \mathrm{~F}^{+}(t) k_{2}^{+}(t) \cdot \mathbb{X} .
\end{aligned}
$$

Applying (4.5) to the second term in the right hand side of (4.2) we obtain

$$
\begin{aligned}
\mathcal{T}_{2}(t) \cdot P_{f}^{-}\left(F\left(t_{n}\right)\right) \cdot F\left(t_{n-1}\right) & \cdots F\left(t_{1}\right) \\
& \sim_{I} \frac{\left(q-q^{-1}\right) t_{n} / t}{1-t_{n} / t} \mathrm{~F}^{+}(t) k_{2}^{+}(t) \cdot F\left(t_{n-1}\right) \cdots F\left(t_{1}\right) .
\end{aligned}
$$

Since any equality modulo ideal $I$ implies the related equality modulo all the ideals $I, J$ and $K$, the relations (4.4) and (4.6) imply the statement of Proposition 4.2. 
Corollary 4.3. Relation (4.1) can be considered as the equality

$$
\begin{aligned}
\mathcal{T}_{2}(t) & P_{f}^{+}(\mathcal{F}(\bar{t})) \\
\sim_{J} & P_{f}^{+}(\mathcal{F}(\bar{t}))\left(\prod_{i=1}^{n} \frac{q^{-1}-q t_{i} / t}{1-t_{i} / t} k_{1}^{+}(t)+\prod_{i=1}^{n} \frac{q-q^{-1} t_{i} / t}{1-t_{i} / t} k_{2}^{+}(t)\right) \\
& +n \overline{\operatorname{Sym}}_{\bar{t}}\left(P_{f}^{+}\left(\mathrm{F}^{+}(t) k_{2}^{+}(t) F\left(t_{n}\right) \cdots F\left(t_{2}\right)\right) \frac{\left(q-q^{-1}\right) t_{1} / t}{1-t_{1} / t} \psi^{+}\left(t_{1}\right)\right) \\
& -n \overline{\operatorname{Sym}}_{\bar{t}}\left(P_{f}^{+}\left(\mathrm{F}^{+}(t) k_{2}^{+}(t) F\left(t_{n-1}\right) \cdots F\left(t_{1}\right)\right) \frac{\left(q-q^{-1}\right) t_{n} / t}{1-t_{n} / t}\right),
\end{aligned}
$$

in $U_{q}\left(\mathfrak{b}^{+}\right)$modulo elements of the ideal $J$. The sum of the last two terms in the above equality belongs to the left ideal $\mathcal{B}$ (see Definition 2.3).

Proof. Left hand side of the equality (4.1) belongs to $U_{q}\left(\mathfrak{b}^{+}\right)$while the right hand side does not. We can imposing projection $P_{f}^{+}$onto this right hand side to cancel all the terms which belongs to the ideal $I$.

\subsection{Proof of Theorem 1 for $\bar{U}_{q}\left(\widehat{\mathfrak{g l}}_{2}\right)$}

Let us compare the last two lines in (4.1). They contain so called 'unwanted' terms. In order to cancel them we will use the following properties of $q$-symmetrization. For any formal series $G\left(t_{1}, \ldots, t_{n}\right)$ on $n$ formal variables $t_{i}$ we have

$$
\begin{aligned}
n & \overline{\operatorname{Sym}}_{\bar{t}} G\left(t_{1}, \ldots, t_{n}\right) \\
& =\sum_{m=1}^{n} \prod_{j=m+1}^{n} \frac{q-q^{-1} t_{m} / t_{j}}{q^{-1}-q t_{m} / t_{j}} \overline{\operatorname{Sym}}_{\bar{t} t_{m}} G\left(t_{1}, \ldots, t_{m-1}, t_{m+1}, \ldots, t_{n}, t_{m}\right)
\end{aligned}
$$

and

$$
\begin{aligned}
& n \overline{\operatorname{Sym}}_{\bar{t}} G\left(t_{1}, \ldots, t_{n}\right) \\
& \quad=\sum_{m=1}^{n} \prod_{j=1}^{m-1} \frac{q-q^{-1} t_{j} / t_{m}}{q^{-1}-q t_{j} / t_{m}} \overline{\operatorname{Sym}} \bar{t}_{\backslash t_{m}} G\left(t_{m}, t_{1}, \ldots, t_{m-1}, t_{m+1}, \ldots, t_{n}\right),
\end{aligned}
$$

where $q$-symmetrization in the right hand sides of this formal series identities runs over $(n-1)$ variables $\bar{t} \backslash t_{m}=\left\{t_{1}, \ldots, t_{m-1}, t_{m+1}, \ldots, t_{m}\right\}$. Note that formulas (4.7) and (4.8) are particular cases of the property (3.17) for $s=n$ and $s=1$, respectively.

Using formulas (4.7) and (4.8) we may write the difference of unwanted terms in (4.1) as a sum

$$
\begin{aligned}
n \sum_{m=1}^{n} \overline{\operatorname{Sym}}_{\bar{t} \backslash t_{m}} & \left(\mathrm{~F}^{+}(t) k_{2}^{+}(t) F\left(t_{n}\right) \cdots F\left(t_{m+1}\right) F\left(t_{m-1}\right) \cdots F\left(t_{1}\right) \frac{\left(q-q^{-1}\right) t_{m} / t}{1-t_{m} / t}\right. \\
& \left.\left(\prod_{j=m+1}^{n} \frac{q-q^{-1} t_{m} / t_{j}}{q^{-1}-q t_{m} / t_{j}} \psi^{+}\left(t_{m}\right)-\prod_{j=1}^{m-1} \frac{q-q^{-1} t_{j} / t_{m}}{q^{-1}-q t_{j} / t_{m}}\right)\right) .
\end{aligned}
$$


Applying to this equality the projection $P_{f}^{+}$, as it was explained in the proof of Corrolary 4.3 , we obtain the elements from the left ideal $\mathcal{B}$. Latter elements are vanishing if the Bethe parameters satisfy the universal Bethe equations [1]

$$
\psi^{+}\left(t_{m}\right)=\frac{k_{1}^{+}\left(t_{m}\right)}{k_{2}^{+}\left(t_{m}\right)}=\prod_{j \neq m}^{n} \frac{q-q^{-1} t_{j} / t_{m}}{q^{-1}-q t_{j} / t_{m}}, \quad m=1, \ldots, n .
$$

Thus, Theorem 1 in the case $N=2$ is proved.

\subsection{General case}

4.3.1. Preliminary exchange relations. To consider the general case of Theorem 1 , we need the embedding $\Psi: \bar{U}_{q}\left(\widehat{\mathfrak{g l}}_{N}\right) \hookrightarrow \bar{U}_{q}\left(\widehat{\mathfrak{g l}}_{N+1}\right)$, defined by the rule ${ }^{4}$

$$
\Psi\left(\mathrm{L}_{i, j}^{[N]}(t)\right)=\mathrm{L}_{i+1, j+1}(t), \quad i, j=1, \ldots, N .
$$

Let $\mathcal{T}_{N}^{\prime}(t)=\sum_{i=2}^{N+1} L_{i i}(t)$ be the universal transfer matrix for the algebra $\bar{U}_{q}\left(\widehat{\mathfrak{g l}}_{N}\right)$ and $\mathcal{W}_{N}^{\prime}\left(\bar{t}_{\left[\bar{n}^{\prime}\right]}\right)$ be the universal weight function, where $\bar{n}^{\prime}$ is a set of the positive integers $\left\{n_{2}, \ldots, n_{N}\right\}$ and $\bar{t}_{\left[\bar{n}^{\prime}\right]}$ is an associated set of the formal variables:

$$
\bar{t}_{\left[\bar{n}^{\prime}\right]}=\left\{t_{1}^{2}, \ldots, t_{n_{2}}^{2} ; \ldots ; t_{1}^{N}, \ldots, t_{n_{N}}^{N}\right\} .
$$

Using the result of Corollary 3.6 we present the $\bar{U}_{q}\left(\widehat{\mathfrak{g l}}_{N+1}\right)$-universal weight function $\mathcal{W}_{N+1}\left(\bar{t}_{[\bar{n}]}\right)$ in the form

$$
\mathcal{W}_{N+1}\left(\bar{t}_{[\bar{n}]}\right)=\mathcal{W}_{N}^{\prime}\left(\bar{t}_{\left[\bar{n}^{\prime}\right]}\right) \cdot \mathcal{F}\left(\bar{t}_{\left[n_{1}\right]}\right)-\mathbb{S},
$$

where $\mathbb{S}$ contains the sum of terms as in the right hand side of (3.32) and the redundant terms $\mathbb{W}$ such that $: \mathcal{T}_{N+1}(t) \cdot \mathbb{W}: \sim_{I} 0$.

We consider the product

$$
\mathcal{T}_{N+1}(t) \cdot \mathcal{W}_{N+1}\left(\bar{t}_{[\bar{n}]}\right)=\mathcal{T}_{N+1}(t) \cdot \mathcal{W}_{N}^{\prime}\left(\bar{t}_{\left[\bar{n}^{\prime}\right]}\right) \cdot \mathcal{F}\left(\bar{t}_{\left[n_{1}\right]}\right)-\mathcal{T}_{N+1}(t) \cdot \mathbb{S} .
$$

Since $\mathcal{T}_{N+1}(t)=\mathrm{L}_{11}(t)+\mathcal{T}_{N}^{\prime}(t)$ we rewrite the first term in the right hand side of (4.12) modulo terms from the ideal $I$ :

$$
\mathcal{T}_{N}^{\prime}(t) \cdot \mathcal{W}_{N}^{\prime}\left(\bar{t}_{\left[\bar{n}^{\prime}\right]}\right) \cdot \mathcal{F}\left(\bar{t}_{\left[n_{1}\right]}\right)+\mathrm{L}_{11}(t) \cdot \mathcal{F}\left(\bar{t}_{[\bar{n}]}\right) .
$$

To obtain (4.13) we use the result of the following

Lemma 4.4.

$$
\mathrm{L}_{11}(t) \cdot \mathcal{W}_{N}^{\prime}\left(\bar{t}_{\left[\bar{n}^{\prime}\right]}\right) \cdot \mathcal{F}\left(\bar{t}_{\left[n_{1}\right]}\right) \sim_{I} \mathrm{~L}_{11}(t) \cdot \mathcal{F}\left(\bar{t}_{[\bar{n}]}\right)
$$

\footnotetext{
${ }^{4}$ We omit writing explicitly superscript ' + ' of the L-operator and their Gauss coordinate, assuming that they are always from the standard positive Borel subalgebra $U_{q}\left(\mathfrak{b}^{+}\right)$.
} 
Proof. The universal Bethe vector $\mathcal{W}_{N}^{\prime}\left(\bar{t}_{\left[\bar{n}^{\prime}\right]}\right)$ for the algebra $\bar{U}_{q}\left(\widehat{\mathfrak{g l}}_{N}\right)$ embedded into $\bar{U}_{q}\left(\widehat{\mathfrak{g l}}_{N+1}\right)$ by (4.10) depends on the modes of the currents $F_{2}(t), \ldots, F_{N}(t)$ and is given by the projection $P_{f}^{+}\left(\mathcal{F}\left(\bar{t}_{\left[\bar{n}^{\prime}\right]}\right)\right)$ of the product of these currents. According to the ordering rules (3.21) we may replace this projection by the product of the total currents $\mathcal{F}\left(\bar{t}_{\left[\bar{n}^{\prime}\right]}\right)$ subtracting the terms which have on the left the negative projection of the currents $P_{f}^{-}\left(F_{c, d}(t)\right)$ with $2 \leq d<c \leq N+1$. According to (3.41) the product $\mathrm{L}_{11}(t) P_{f}^{-}\left(F_{c, d}\left(t^{\prime}\right)\right) \sim_{I} 0$. This proves the statement of the lemma.

Now we apply the result of Proposition 3.9 to the second term in the right hand side of (4.12). We have

$$
\begin{aligned}
\mathcal{T}_{N+1}(t) \cdot \mathbb{S} \sim_{I} & \overline{\operatorname{Sym}}_{\bar{t}_{[\bar{n}]}}\left(\sum_{m=1}^{N} \mathrm{~L}_{1, m+1}(t) \cdot P_{f}^{+}\left(\mathcal{F}\left(\bar{t}_{\left[\bar{n}^{\prime}\right]_{m}}\right)\right) \cdot \mathcal{F}\left(\bar{t}_{\left[n_{1}-1\right]}^{1}\right)\right. \\
& \left.\times \prod_{a=1}^{m}\left(n_{a}\right) \frac{\left(q-q^{-1}\right)^{m} t_{n_{1}}^{1} / t}{1-t_{n_{1}}^{1} / t} \mathbb{Z}_{m}\left(\bar{t}_{[\bar{n}]}\right)\right)
\end{aligned}
$$

where the sets of the formal variables $\bar{t}_{\left[\bar{n}^{\prime}\right]_{m}}$ are defined by (3.31) and a rational series $\mathbb{Z}_{m}\left(\bar{t}_{[\bar{n}]}\right)$ is defined by (3.33). We can simplify the right hand side of $(4.15)$ replacing the projection $P_{f}^{+}\left(\mathcal{F}\left(\bar{t}_{\left[\bar{n}^{\prime}\right]_{m}}\right)\right)$ by the product of the currents $\mathcal{F}\left(\bar{t}_{\left[\bar{n}^{\prime}\right]_{m}}\right)$ due to the following

\section{Lemma 4.5.}

$$
\mathrm{L}_{1, m+1}(t) \cdot P_{f}^{+}\left(\mathcal{F}\left(\bar{t}_{\left[\bar{n}_{m}^{\prime}\right]}\right)\right) \sim_{I} \mathrm{~L}_{1, m+1}(t) \cdot \mathcal{F}\left(\bar{t}_{\left[\bar{n}_{m}^{\prime}\right]}\right)
$$

Proof of this lemma is analogous to the proof of Lemma 4.4.

Using the explicit form of the matrix elements $\mathrm{L}_{1,1}(t)$ and $\mathrm{L}_{1, m+1}(t)$ in terms of the Gauss coordinates

$$
\begin{aligned}
\mathrm{L}_{1,1}(t) & =k_{1}^{+}(t)+\sum_{j=2}^{N+1} \mathrm{~F}_{j, 1}^{+}(t) k_{j}^{+}(t) \mathrm{E}_{1, j}^{+}(t), \\
\mathrm{L}_{1, m+1}(t) & =\mathrm{F}_{m+1,1}^{+}(t) k_{m+1}^{+}(t)+\sum_{j=m+2}^{N+1} \mathrm{~F}_{j, 1}^{+}(t) k_{j}^{+}(t) \mathrm{E}_{m+1, j}^{+}(t),
\end{aligned}
$$

we can present the product in the left hand side of (4.12) as the sum of the terms modulo ideal $I$

$$
\begin{aligned}
& \mathcal{T}_{N+1}(t) \cdot \mathcal{W}_{N+1}\left(\bar{t}_{[\bar{n}]}\right) \sim_{I} \mathcal{T}_{N}^{\prime}(t) \cdot \mathcal{W}_{N}^{\prime}\left(\bar{t}_{\left[\bar{n}^{\prime}\right]}\right) \cdot \mathcal{F}\left(\bar{t}_{\left[n_{1}\right]}\right)+k_{1}^{+}(t) \cdot \mathcal{F}\left(\bar{t}_{[\bar{n}]}\right) \\
& \quad+\sum_{j=2}^{N+1} \mathrm{~F}_{j, 1}^{+}(t) k_{j}^{+}(t)\left(\mathrm{E}_{1, j}^{+}(t) \cdot \mathcal{F}\left(\bar{t}_{[\bar{n}]}\right)-\mathbb{A}_{j-1}-\sum_{m=1}^{j-2} \mathrm{E}_{m+1, j}^{+}(t) \cdot \mathbb{A}_{m}\right)
\end{aligned}
$$


where

$$
\mathbb{A}_{m}=\left(q-q^{-1}\right)^{m} \prod_{a=1}^{m}\left(n_{a}\right) \overline{\operatorname{Sym}}_{\bar{t}_{[\bar{n}]}}\left(\mathcal{F}\left(\bar{t}_{\left[\bar{n}^{\prime}\right]_{m}}\right) \cdot \mathcal{F}\left(\bar{t}_{\left[n_{1}-1\right]}^{1}\right) \cdot \mathbb{Z}_{m}\left(\bar{t}_{[\bar{n}]}\right) \frac{t_{n_{1} / t}^{1} / t}{1-t_{n_{1}}^{1} / t}\right) .
$$

Calculation of (4.17) modulo elements of the ideal $I$ is useful since now to continue ordering we have to exchange the Gauss coordinates $\mathrm{E}_{m+1, j}^{+}$with total currents. These exchange relations are based on the formulae given by Lemma 3.4:

$$
\mathrm{E}_{m+1, j}^{+}(t)=\left(q-q^{-1}\right)^{m+2-j} \hat{S}_{m+1} \hat{S}_{m+2} \cdots \hat{S}_{j-2}\left(\mathrm{E}_{j-1, j}^{+}(t)\right),
$$

where the screening operators is defined as $\hat{S}_{i}(B)=E_{i}[0] B-q B E_{i}[0]$, the formulae

$$
\left[\mathrm{E}_{i, i+1}^{+}(t), F_{j}\left(t^{\prime}\right)\right] \sim_{K} \delta_{i, j} \frac{\left(q-q^{-1}\right) t^{\prime} / t}{1-t^{\prime} / t} \psi_{i}^{+}\left(t^{\prime}\right),
$$

and

$$
\left[E_{i}[0], F_{j}\left(t^{\prime}\right)\right] \sim_{K} \delta_{i, j}\left(q-q^{-1}\right) \psi_{i}^{+}\left(t^{\prime}\right) .
$$

Here $\psi_{i}^{+}(t)=k_{i}^{+}(t) k_{i+1}^{+}(t)^{-1}$. Recall that the symbol ' $\sim_{K}$ ' means an equality modulo terms of the ideal $K$ which composed from the elements of $\bar{U}_{q}\left(\widehat{\mathfrak{g l}}_{N}\right)$ with any mode of the negative Cartan current $\psi_{i}^{-}(t)=k_{i}^{-}(t) k_{i+1}^{-}(t)^{-1}$.

The ordering of the first term for $j=2$ in the right hand side of (4.17) can be performed as in the case of the algebra $U_{q}\left(\widehat{\mathfrak{s l}}_{2}\right)$ since the Gauss coordinate $\mathrm{E}_{1,2}^{+}(t)$ does not commute only with the currents $F_{1}\left(t_{\ell}^{1}\right)$. This term is equal to (modulo elements from the ideals $K$ and $J$ )

$$
\begin{array}{r}
n_{1}\left(q-q^{-1}\right) \mathrm{F}_{2,1}^{+}(t) k_{2}^{+}(t) \overline{\operatorname{Sym}}_{\bar{t}_{[\bar{n}]}}\left(\mathcal{F}\left(\bar{t}_{\left[\bar{n}^{\prime}\right]_{1}}\right) \cdot \mathcal{F}\left(\bar{t}_{\left[1, n_{1}\right]}^{1}\right) \frac{t_{1}^{1} / t}{1-t_{1}^{1} / t} \psi_{1}^{+}\left(t_{1}^{1}\right)\right. \\
\left.-\mathcal{F}\left(\bar{t}_{\left[\bar{n}^{\prime}\right]_{1}}\right) \cdot \mathcal{F}\left(\bar{t}_{\left[n_{1}-1\right]}^{1}\right) \frac{t_{n_{1}}^{1} / t}{1-t_{n_{1}}^{1} / t} \prod_{j=1}^{n_{2}} \frac{q-q^{-1} t_{n_{1}}^{1} / t_{j}^{2}}{1-t_{n_{1}}^{1} / t_{j}^{2}}\right) .
\end{array}
$$

The ordering of the Gauss coordinates $\mathrm{E}_{m+1, j}^{+}(t)$ with total currents is more involved. To perform this ordering we have to use besides (4.19), (4.20), (4.21) also the relation

$$
\hat{S}_{i}\left(\psi_{i+1}^{+}(t)\right)=E_{i}[0] \psi_{i+1}^{+}(t)-q \psi_{i+1}^{+}(t) E_{i}[0]=\left(q-q^{-1}\right) \psi_{i+1}^{+}(t) \mathrm{E}_{i}^{+}(t) .
$$

Fix the $j$ th term of the sum in the right hand side of (4.17) and denote

$$
\mathbb{R}_{j}=\mathrm{E}_{1, j}^{+}(t) \cdot \mathcal{F}\left(\bar{t}_{[\bar{n}]}\right)-\left(q-q^{-1}\right)^{j-1} \mathbb{A}_{j-1}-\sum_{m=1}^{j-2}\left(q-q^{-1}\right)^{m} \mathrm{E}_{m+1, j}^{+}(t) \cdot \mathbb{A}_{m}
$$

We will exchange the Gauss coordinates $\mathrm{E}_{m+1, j}^{+}(t)$ with total current. As above we will calculate modulo elements of the ideals $K$ and $J$.

We will describe this calculation as the sequence of the steps. 
1. According to (4.19) the Gauss coordinate $\mathrm{E}_{m+1, j}^{+}(t)$ is composed from the zero mode of the currents $E_{i}(t)$ with $i=m+1, \ldots, j-2$ and the Gauss coordinate $\mathrm{E}_{j-1, j}^{+}(t)$. It means that this Gauss coordinate will have nontrivial commutation relations only with the currents $F_{a}\left(t_{s}^{a}\right)$ for $a=m+1, \ldots, j-1$. The move of the Gauss coordinates $\mathrm{E}_{m+1, j}^{+}(t)$ through the total current $F_{a}\left(t_{s}^{a}\right)$ with $a=m+1, \ldots, j-2$ produces the terms which belong to the ideal $J$. These terms appear after commuting zero modes $E_{a}[0]$ with the current $F_{a}\left(t_{s}^{a}\right)$ and can be neglected since we perform the calculation modulo elements of the ideal $J$. The nontrivial terms, which we will consider, are created after exchanging the Gauss coordinate $\mathrm{E}_{m+1, j}^{+}(t)$ with the currents $F_{j-1}\left(t_{s}^{j-1}\right)$. At the first step the Cartan currents $\psi_{j-1}^{+}\left(t_{s}^{j-1}\right)$ together with a rational factor $\frac{\left(q-q^{-1}\right) t_{1}^{j-1} / t}{1-t_{1}^{j-1} / t}$ appear according to (4.20) after an exchange of $\mathrm{E}_{j-1, j}^{+}(t)$ and the total current $F_{j-1}\left(t_{s}^{j-1}\right)$ in the place of the latter current. This Cartan current should be moved to the right of the product of type $j-1$ currents. All the terms resulting from the exchange relation of $\mathrm{E}_{j-1, j}^{+}(t)$ and $F_{j-1}\left(t_{s}^{j-1}\right)$ can be presented as the $q$-symmetrization over the type $j-1$ formal variables of the single term which appears after commutation of $\mathrm{E}_{j-1, j}^{+}(t)$ with $F_{j-1}\left(t_{1}^{j-1}\right)$. Due to properties (3.16) this $q$-symmetrization is absorbed into 'global' $q$-symmetrization entering into definitions of the elements $\mathbb{A}_{m}$ (4.18) producing factor $n_{j-1}$ due to definition of the $q$-symmetrization. The product of the current $\mathcal{F}\left(\bar{t}_{[\bar{n}]}\right)$ in (4.24) can be also presented as the 'global' $q$-symmetrization over the set of the variables $\bar{t}_{[\bar{n}]}$.

2. The sequence of the screening operators $\hat{S}_{m+1} \cdots \hat{S}_{j-2}$ which enter the formula for the Gauss coordinate $\mathrm{E}_{m+1, j}^{+}(t)$ are applied according to equation (4.23) to replace the Cartan current $\psi_{j-1}^{+}\left(t_{1}^{j-1}\right)$ on the right of the product of $j$ - 1-type currents by the factor $\psi_{j-1}^{+}\left(t_{1}^{j-1}\right) \mathrm{E}_{m+1, j-1}^{+}\left(t_{1}^{j-1}\right)$.

3. The Gauss coordinate $\mathrm{E}_{m+1, j-1}^{+}\left(t_{1}^{j-1}\right)$ should be moved through the total currents of type $j-2$ to produce $q$-symmetrization over variables of the same type and the factor $\psi_{j-2}^{+}\left(t_{1}^{j-2}\right) \mathrm{E}_{m+1, j-2}^{+}\left(t_{1}^{j-2}\right)$ on the right of the product of this type current accompanied with a rational factor $\frac{\left(q-q^{-1}\right) t_{1}^{j-2} / t_{1}^{j-1}}{1-t_{1}^{j-2} / t_{1}^{j-1}}$. Note that the factor $n_{j-2}$ appears also due to definition of the $q$-symmetrization.

4. After moving all Gauss coordinates $\mathrm{E}_{m+1, a}^{+}\left(t_{1}^{a}\right)$ to the right we are left with a product of total currents where all the currents $F_{a}\left(t_{1}^{a}\right)$ have been replaced by the Cartan currents $\psi_{a}^{+}\left(t_{1}^{a}\right)$ with $a=m+1, \ldots, j-1$ together with the rational factors $n_{a} \frac{\left(q-q^{-1}\right) t_{1}^{a} / t_{1}^{a+1}}{1-t_{1}^{a} / t_{1}^{a+1}}$. Then, we have to move all these Cartan currents to the right of all total currents producing the rational factors of the Bethe parameters. 
These calculations result to the following exchange relations of the Gauss coordinate $\mathrm{E}_{m+1, j}^{+}(t)$ and the product of the currents $\mathcal{F}\left(\bar{t}_{\left[\bar{n}^{\prime}\right]_{m}}\right) \cdot \mathcal{F}\left(\bar{t}_{\left[n_{1}-1\right]}^{1}\right)$

$$
\begin{aligned}
& \mathrm{E}_{m+1, j}^{+}(t) \mathcal{F}\left(\bar{t}_{\left[\bar{n}^{\prime}\right]_{m}}\right) \cdot \mathcal{F}\left(\bar{t}_{\left[n_{1}-1\right]}^{1}\right) \\
& \sim_{J, K}\left(q-q^{-1}\right)^{j-m-1} \prod_{a=m+1}^{j-1}\left(n_{a}\right) \overline{\operatorname{Sym}}_{\bar{t}_{[\bar{n}]}}\left(\mathcal{F}\left(\bar{t}_{\left[n_{N}\right]}^{N}\right) \cdots \mathcal{F}\left(\bar{t}_{\left[n_{j}\right]}^{j}\right)\right. \\
& \quad \times \mathcal{F}\left(\bar{t}_{\left[1, n_{j-1}\right]}^{j-1}\right) \cdots \mathcal{F}\left(\bar{t}_{\left[1, n_{m+1}\right]}^{m+1}\right) \mathcal{F}\left(\bar{t}_{\left[n_{m}-1\right]}^{m}\right) \cdots \mathcal{F}\left(\bar{t}_{\left[n_{1}-1\right]}^{1}\right) \\
& \left.\quad \times \frac{t_{1}^{j-1} / t}{1-t_{1}^{j-1} / t} \mathbb{Y}_{m}\left(\bar{t}_{[\bar{n}]}\right) \prod_{a=m+1}^{j-1} \psi_{a}^{+}\left(t_{1}^{a}\right)\right)
\end{aligned}
$$

where a rational series $\mathbb{Y}_{m}\left(\bar{t}_{[\bar{n}]}\right)$ is defined by the relation

$$
\mathbb{Y}_{m}\left(\bar{t}_{[\bar{n}]}\right)=\prod_{a=m+1}^{j-2}\left(\frac{t_{1}^{a} / t_{1}^{a+1}}{1-t_{1}^{a} / t_{1}^{a+1}} \prod_{k=2}^{n_{a}} \frac{q-q^{-1} t_{k}^{a} / t_{1}^{a+1}}{1-t_{k}^{a} / t_{1}^{a+1}}\right) \prod_{k=1}^{n_{m}-1} \frac{q-q^{-1} t_{k}^{m} / t_{1}^{m+1}}{1-t_{k}^{m} / t_{1}^{m+1}}
$$

Recall that the notation $\bar{t}_{\left[1, n_{a}\right]}^{a}$ means the collection of the variables $\left\{t_{2}^{a}, \ldots, t_{n_{a}}^{a}\right\}$.

We get finally the following presentation of the product $\mathcal{T}_{N+1}(t) \cdot \mathcal{W}_{N+1}\left(\bar{t}_{[\bar{n}]}\right)$ :

$$
\begin{aligned}
\mathcal{T}_{N+1}(t) \cdot \mathcal{W}_{N+1}\left(\bar{t}_{[\bar{n}]}\right) \approx & \mathcal{T}_{N}^{\prime}(t) \cdot \mathcal{W}_{N}^{\prime}\left(\bar{t}_{\left[\bar{n}^{\prime}\right]}\right) \cdot \mathcal{F}\left(\bar{t}_{\left[n_{1}\right]}\right) \\
& +\mathcal{W}_{N+1}\left(\bar{t}_{[\bar{n}]}\right) \cdot k_{1}^{+}(t) \prod_{i=1}^{n_{1}} \frac{q^{-1}-q t_{i}^{1} / t}{1-t_{i}^{1} / t} \prod_{i=1}^{n_{2}} \frac{q-q^{-1} t_{i}^{2} / t}{1-t_{i}^{2} / t} \\
& +\sum_{j=2}^{N+1}\left(q-q^{-1}\right)^{j-1} \prod_{a=1}^{j-1}\left(n_{a}\right) \mathrm{F}_{j, 1}^{+}(t) k_{j}^{+}(t) \cdot \mathbb{R}_{j},
\end{aligned}
$$

where the symbol ' $\approx$ ' means an equality modulo the ideals $I, J$ and $K$ (see Definition 4.1). The elements $\mathbb{R}_{j}$ have the structure

$$
\begin{aligned}
\mathbb{R}_{j} & =\overline{\operatorname{Sym}}_{\bar{t}_{[\bar{n}]}}\left(\mathcal{F}\left(\bar{t}_{\left[n_{N}\right]}^{N}\right) \cdots \mathcal{F}\left(\bar{t}_{\left[n_{j}\right]}^{j}\right) \mathcal{F}\left(\bar{t}_{\left[1, n_{j-1}\right]}^{j-1}\right) \cdots \mathcal{F}\left(\bar{t}_{\left[1, n_{1}\right]}^{1}\right) \mathbb{Y}_{0}\left(\bar{t}_{[\bar{n}]}\right) \frac{t_{1}^{j-1} / t}{1-t_{1}^{j-1} / t}\right. \\
& \prod_{a=1}^{j-1} \psi_{a}^{+}\left(t_{1}^{a}\right)-\mathcal{F}\left(\bar{t}_{\left[n_{N}\right]}^{N}\right) \cdots \mathcal{F}\left(\bar{t}_{\left[n_{j}\right]}^{j}\right) \mathcal{F}\left(\bar{t}_{\left[n_{j-1}-1\right]}^{j-1}\right) \cdots \mathcal{F}\left(\bar{t}_{\left[n_{1}-1\right]}^{1}\right) \cdot \mathbb{Z}_{j-1}\left(\bar{t}_{[\bar{n}]}\right) \frac{t_{n_{1} / t}^{1}}{1-t_{n_{1}}^{1} / t} \\
& -\sum_{m=1}^{j-2}\left(\mathcal{F}\left(\bar{t}_{\left[n_{N}\right]}^{N}\right) \cdots \mathcal{F}\left(\bar{t}_{\left[n_{j}\right]}^{j}\right) \mathcal{F}\left(\bar{t}_{\left[1, n_{j-1}\right]}^{j-1}\right) \cdots \mathcal{F}\left(\bar{t}_{\left[1, n_{m+1}\right]}^{m+1}\right) \mathcal{F}\left(\bar{t}_{\left[n_{m}-1\right]}^{m}\right) \cdots \mathcal{F}\left(\bar{t}_{\left[n_{1}-1\right]}^{1}\right)\right. \\
& \left.\left.\times \frac{t_{n_{1}}^{1} / t}{1-t_{n_{1}}^{1} / t} \frac{t_{1}^{j-1} / t}{1-t_{1}^{j-1} / t} \mathbb{Z}_{m}\left(\bar{t}_{\left[\bar{n}_{]}\right]}\right) \mathbb{Y}_{m}\left(\bar{t}_{[\bar{n}]}\right) \prod_{a=m+1}^{j-1} \psi_{a}^{+}\left(t_{1}^{a}\right)\right)\right) .
\end{aligned}
$$

The series $\mathbb{Z}_{m}\left(\bar{t}_{[\bar{n}]}\right)$ is defined by the relation (3.33). 
4.3.2. Proof of Theorem 1. The following proposition generalizes Proposition 4.2 and serves as a main statement which is proved by induction over $N$.

Proposition 4.6. There is a formal series equality in the algebra $\bar{U}_{q}\left(\widehat{\mathfrak{g l}}_{N}\right)$

$$
\begin{aligned}
\mathcal{T}_{N}(t) & \cdot \mathcal{W}_{N}\left(\bar{t}_{[\bar{n}]}\right) \approx \mathcal{W}_{N}\left(\bar{t}_{[\bar{n}]}\right) \cdot\left(\sum_{a=1}^{N} k_{a}^{+}(t) \prod_{k=1}^{n_{a-1}} \frac{q-q^{-1} t_{k}^{a-1} / t}{1-t_{k}^{a-1} / t} \prod_{k=1}^{n_{a}} \frac{q^{-1}-q t_{k}^{a} / t}{1-t_{k}^{a} / t}\right) \\
& +\overline{\operatorname{Sym}}_{\bar{t}_{[\bar{n}]}}\left(\sum _ { j = 2 } ^ { N } \prod _ { a = 1 } ^ { j - 1 } ( n _ { a } ) \left(\left(q-q^{-1}\right)^{j-1} \mathrm{~F}_{j, 1}^{+}(t) k_{j}^{+}(t) \cdot \mathcal{F}\left(\bar{t}_{\left[n_{N-1}\right]}^{N-1}\right) \cdots \mathcal{F}\left(\bar{t}_{\left[n_{j}\right]}^{j}\right)\right.\right. \\
& \left.\times \mathcal{F}\left(\bar{t}_{\left[n_{j-1}-1\right]}^{j-1}\right) \cdots \mathcal{F}\left(\bar{t}_{\left[n_{1}-1\right]}^{1}\right) \cdot \mathbb{Z}_{j-1}\left(\bar{t}_{[\bar{n}]}\right) \frac{t_{n_{j-1}}^{j-1} / t}{1-t_{n_{j-1}}^{j-1} / t}\right) \\
& \left.\times\left(\psi_{1}^{+}\left(t_{n_{1}}^{1}\right) \prod_{k=1}^{n_{1}-1} \frac{q^{-1}-q t_{k}^{1} / t_{n_{1}}^{1}}{q-q^{-1} t_{k}^{1} / t_{n_{1}}^{1}} \prod_{k=1}^{n_{2}} \frac{1-t_{k}^{2} / t_{n_{1}}^{1}}{q^{-1}-q t_{k}^{2} / t_{n_{1}}^{1}}-1\right)\right) \\
\sim_{\mathcal{B}} & \mathcal{W}_{N}\left(\bar{t}_{\left[\bar{n}_{]}\right]}\right) \cdot\left(\sum_{a=1}^{N} k_{a}^{+}(t) \prod_{k=1}^{n_{a-1}} \frac{q-q^{-1} t_{k}^{a-1} / t}{1-t_{k}^{a-1} / t} \prod_{k=1}^{n_{a}} \frac{q^{-1}-q t_{k}^{a} / t}{1-t_{k}^{a} / t}\right)
\end{aligned}
$$

if the set $\left\{t_{j}^{i}\right\}$ of the Bethe parameters satisfies the set of the universal Bethe equations, $i=2, \ldots, N-1, j=1, \ldots, n_{i}$ :

$$
\frac{k_{i}^{+}\left(t_{j}^{i}\right)}{k_{i+1}^{+}\left(t_{j}^{i}\right)}=\prod_{m \neq j}^{n_{i}} \frac{q-q^{-1} t_{m}^{i} / t_{j}^{i}}{q^{-1}-q t_{m}^{i} / t_{j}^{i}} \prod_{m=1}^{n_{i-1}} \frac{1-t_{m}^{i-1} / t_{j}^{i}}{q-q^{-1} t_{m}^{i-1} / t_{j}^{i}} \prod_{m=1}^{n_{i+1}} \frac{q^{-1}-q t_{m}^{i+1} / t_{j}^{i}}{1-t_{m}^{i+1} / t_{j}^{i}}
$$

Proof. We will proof this proposition by induction over $N$ taking as the base of the induction the statement of Proposition 4.2. We assume that the equality (4.28) is valid for the algebra $\bar{U}_{q}\left(\widehat{\mathfrak{g l}}_{N}\right)$ embedded into $\bar{U}_{q}\left(\widehat{\mathfrak{g l}}_{N+1}\right)$ by the relation (4.10) and prove from the relation (4.26) that (4.28) is valid also for the algebra $\bar{U}_{q}\left(\widehat{\mathfrak{g l}}_{N+1}\right)$.

First we rewrite the induction assumption (4.28) for the algebra $\bar{U}_{q}\left(\widehat{\mathfrak{g l}}_{N}\right)$ embedded into $\bar{U}_{q}\left(\widehat{\mathfrak{g l}}_{N+1}\right)$ by (4.10). It takes the form

$$
\begin{aligned}
& \mathcal{T}_{N}^{\prime}(t) \cdot \mathcal{W}_{N}^{\prime}\left(\bar{t}_{\left[\bar{n}^{\prime}\right]}\right) \\
& \quad \approx \mathcal{W}_{N}^{\prime}\left(\bar{t}_{\left[\bar{n}^{\prime}\right]}\right) \cdot\left(\sum_{a=2}^{N+1} k_{a}^{+}(t) \prod_{k=1}^{n_{a-1}} \frac{q-q^{-1} t_{k}^{a-1} / t}{1-t_{k}^{a-1} / t} \prod_{k=1}^{n_{a}} \frac{q^{-1}-q t_{k}^{a} / t}{1-t_{k}^{a} / t}\right)+\mathbb{Q}
\end{aligned}
$$


where in 'unwanted' terms

$$
\begin{aligned}
\mathbb{Q}= & \overline{\operatorname{Sym}}_{\bar{t}_{[\bar{n}]}}\left(\sum _ { j = 3 } ^ { N + 1 } \left(\left(q-q^{-1}\right)^{j-1} \prod_{a=2}^{j-1}\left(n_{a}\right) \mathrm{F}_{j, 2}^{+}(t) k_{j}^{+}(t) \cdot \mathcal{F}\left(\bar{t}_{\left[n_{N}\right]}^{N}\right) \cdots \mathcal{F}\left(\bar{t}_{\left[n_{j}\right]}^{j}\right)\right.\right. \\
& \left.\times \mathcal{F}\left(\bar{t}_{\left[n_{j-1}-1\right]}^{j-1}\right) \cdots \mathcal{F}\left(\bar{t}_{\left[n_{2}-1\right]}^{2}\right) \cdot \mathbb{Z}_{j-1}\left(\bar{t}_{\left[\bar{n}^{\prime}\right]}\right) \frac{t_{n_{j-1} / t}^{j-1}}{1-t_{n_{j-1} / t}^{j-1}}\right) \\
& \left.\times\left(\psi_{2}^{+}\left(t_{n_{2}}^{2}\right) \prod_{k=1}^{n_{2}-1} \frac{q^{-1}-q t_{k}^{2} / t_{n_{2}}^{2}}{q-q^{-1} t_{k}^{2} / t_{n_{2}}^{2}} \prod_{k=1}^{n_{3}} \frac{1-t_{k}^{3} / t_{n_{2}}^{2}}{q^{-1}-q t_{k}^{3} / t_{n_{2}}^{2}}-1\right)\right)
\end{aligned}
$$

parameters $\bar{t}_{\bar{n}_{a}}^{a}$ with $a=3, \ldots, N$ satisfy the universal Bethe equations (4.29) for $i=3, \ldots, N$ while parameters $\bar{t}_{\bar{n}_{2}}^{2}$ are free.

We substitute (4.30) into (4.26). First term in the right hand side of (4.30) together with the second term in (4.26) produces 'wanted' terms

$$
\mathcal{W}_{N+1}\left(\bar{t}_{[\bar{n}]}\right) \cdot\left(\sum_{a=1}^{N+1} k_{a}^{+}(t) \prod_{k=1}^{n_{a-1}} \frac{q-q^{-1} t_{k}^{a-1} / t}{1-t_{k}^{a-1} / t} \prod_{k=1}^{n_{a}} \frac{q^{-1}-q t_{k}^{a} / t}{1-t_{k}^{a} / t}\right) .
$$

The terms in the right hand side of (4.30) which belongs to the ideal $J$ will be again in the same ideal after multiplication to the right of $\mathcal{T}_{N}^{\prime}(t) \cdot \mathcal{W}_{N}^{\prime}\left(\bar{t}_{\left[\bar{n}^{\prime}\right]}\right)$ by the product of the first type currents $\mathcal{F}\left(\bar{t}_{\left[n_{1}\right]}\right)$ (see (4.26)). This is because the currents $E_{a}(t)$ commute with the current $F_{1}\left(t^{\prime}\right)$ for $a=2, \ldots, N$.

Fix parameters $\bar{t}_{\bar{n}_{2}}^{2}$ from the condition that the ordered product : $\mathbb{Q} \cdot \mathcal{F}\left(\vec{t}_{\left[n_{1}\right]}\right)$ : of the unwanted terms and the currents of the first type $F_{1}\left(t_{k}^{1}\right)$ belong to the ideal $\mathcal{B}$. This results into Bethe equations

$$
\frac{k_{2}^{+}\left(t_{k}^{2}\right)}{k_{3}^{+}\left(t_{k}^{2}\right)}=\prod_{m \neq k}^{n_{2}} \frac{q-q^{-1} t_{m}^{2} / t_{k}^{2}}{q^{-1}-q t_{m}^{2} / t_{k}^{2}} \prod_{m=1}^{n_{1}} \frac{1-t_{m}^{1} / t_{k}^{2}}{q-q^{-1} t_{m}^{1} / t_{k}^{2}} \prod_{m=1}^{n_{3}} \frac{q^{-1}-q t_{m}^{3} / t_{k}^{2}}{1-t_{m}^{3} / t_{k}^{2}}
$$

for the set of parameters $\bar{t}_{\bar{n}_{2}}^{2}$.

Now we will examine the structure of the terms $\mathbb{R}_{j}$ given by (4.27) by the conditions that parameters $\bar{t}_{\bar{n}_{a}}^{a}$ with $a=2, \ldots, N$ are bounded by the universal Bethe equations (4.29) and (4.32). We replace in $\mathbb{R}_{j}$ the Cartan currents $\psi_{a}^{+}\left(t_{1}^{a}\right)$, $a=2, \ldots, j-1$ by the right hand sides of the universal Bethe equations. Each Bethe equation introduces the factor $\prod_{\ell=2}^{n_{a}} \frac{q t_{1}^{a}-q^{-1} t_{\ell}^{a}}{q^{-1} t_{1}^{a}-q t_{\ell}^{a}}$ under $q$-symmetrization. This allows to use the following property of the $q$-symmetrization, which is a consequence of (4.7) and (4.8):

$$
\overline{\operatorname{Sym}}_{\bar{t}}\left(G\left(t_{1}, t_{2}, \ldots, t_{n}\right) \prod_{\ell=2}^{n} \frac{q^{-1}-q t_{1} / t_{\ell}}{q-q^{-1} t_{1} / t_{\ell}}\right)=\overline{\operatorname{Sym}}_{\bar{t}}\left(G\left(t_{n}, t_{1}, \ldots, t_{n-1}\right)\right)
$$

for arbitrary formal series $G$ of the formal variables $\left(t_{1}, \ldots, t_{n}\right)$. The variables $\left\{t_{1}^{a}, t_{2}^{a}, \ldots, t_{n_{a}}^{a}\right\}$ are replaced by the permuted sets $\left\{t_{n_{a}}^{a}, t_{1}^{a}, \ldots, t_{n_{a}-1}^{a}\right\}$ for $a=$ 
$m+1, \ldots, j-1$. Using an identity

$$
\begin{aligned}
& \frac{1}{t-t_{n_{j-1}}^{j-1}} \prod_{a=1}^{j-2} \frac{1}{t_{n_{a+1}}^{a+1}-t_{n_{a}}^{a}}-\frac{1}{t-t_{n_{1}}^{1}} \prod_{a=1}^{j-2} \frac{1}{t_{n_{a+1}}^{a+1}-t_{n_{a}}^{a}} \\
& -\frac{1}{t-t_{n_{j-1}}^{j-1}} \frac{1}{t-t_{n_{1}}^{1}} \sum_{m=1}^{j-2} \prod_{a=m+1}^{j-2} \frac{1}{t_{n_{a+1}}^{a+1}-t_{n_{a}}^{a}} \prod_{a=1}^{m-1} \frac{1}{t_{n_{a+1}}^{a+1}-t_{n_{a}}^{a}}=0
\end{aligned}
$$

and the explicit forms of the series $\mathbb{Z}_{m}$ and $\mathbb{Y}_{m}$ we observe that the element $\mathbb{R}_{j}$ has the structure

$$
\begin{aligned}
\mathbb{R}_{j}= & \mathcal{F}\left(\bar{t}_{\left[n_{N}\right]}^{N}\right) \cdots \mathcal{F}\left(\bar{t}_{\left[n_{j}\right]}^{j}\right) \cdot \mathcal{F}\left(\bar{t}_{\left[n_{j-1}-1\right]}^{j-1}\right) \cdots \mathcal{F}\left(\bar{t}_{\left[n_{1}-1\right]}^{1}\right) \cdot \mathbb{Z}_{j-1}\left(\bar{t}_{[\bar{n}]}\right) \frac{t_{n_{j-1}}^{j-1} / t}{1-t_{n_{j-1} / t}^{j-1}} \\
& \times\left(\psi_{1}^{+}\left(t_{n_{1}}^{1}\right) \prod_{k=1}^{n_{1}-1} \frac{q^{-1}-q t_{k}^{1} / t_{n_{1}}^{1}}{q-q^{-1} t_{k}^{1} / t_{n_{1}}^{1}} \prod_{k=1}^{n_{2}} \frac{1-t_{k}^{2} / t_{n_{1}}^{1}}{q^{-1}-q t_{k}^{2} / t_{n_{1}}^{1}}-1\right)
\end{aligned}
$$

belongs to the Bethe ideal $\mathcal{B}$ (see Definition 2.3). This proves the statement of the Proposition.

The statement of Theorem 1 follows from Proposition 4.6. The element $\mathbb{R}_{j}$ vanishes if we impose one more universal Bethe equation for the set of the parameters $\left\{\bar{t}_{\left[n_{1}\right]}\right\}$

$$
\frac{k_{1}^{+}\left(t_{j}^{1}\right)}{k_{2}^{+}\left(t_{j}^{1}\right)}=\prod_{m \neq j}^{n_{1}} \frac{q-q^{-1} t_{m}^{1} / t_{j}^{1}}{q^{-1}-q t_{m}^{1} / t_{j}^{1}} \prod_{m=1}^{n_{2}} \frac{q^{-1}-q t_{m}^{2} / t_{j}^{1}}{1-t_{m}^{2} / t_{j}^{1}} .
$$

Since the left hand side and first term in the right hand side of (4.28) belong to the standard Borel subalgebra $U_{q}\left(\mathfrak{b}^{+}\right)$in $\bar{U}_{q}\left(\widehat{\mathfrak{g l}}_{N}\right)$ the equality between them is valid modulo elements of the ideal $J$ and the Bethe ideal $\mathcal{B}$. This finishes the proof of Theorem 1.

\section{Acknowledgements}

This work was partially done when the third author (S.P.) visited Laboratoire d'Annecy-Le-Vieux de Physique Théorique in 2006 and 2007. These visits were possible due to the financial support of the CNRS-Russia exchange program on mathematical physics. He thanks LAPTH for the hospitality and stimulating scientific atmosphere. We are thankful to referee for valuable comments. Work by second (S.K) and third (S.P.) authors was supported in part by RFBR grant 0801-00667 and grant for support of scientific schools NSh-3036.2008.2. Third author (S.P.) was also supported in part by RFBR-CNRS grant 07-02-92166-CNRS. 


\section{References}

[1] D. Arnaudon, N. Crampe, A. Doikou, L. Frappat, E. Ragoucy, Spectrum and Bethe ansatz equations for the $U_{q}(g l(\mathcal{N}))$ closed and open spin chains in any representation, Annales Henri Poincaré 7 (2006), 1217-1268.

[2] J. Ding, I. B. Frenkel, Isomorphism of two realizations of quantum affine algebra $U_{q}\left(\widehat{\mathfrak{g l}}_{N}\right)$, Commun. Math. Phys. 156 (1993), 277-300.

[3] J. Ding, S. Khoroshkin, Weyl group extension of quantized current algebras, Transformation Groups 5 (2000), 35-59.

[4] V. Drinfeld, Quantum groups, In "International Congress of Mathematicians (Berkley, 1986)", Amer. Math. Soc., Providence RI, 1987, 798-820.

[5] V. Drinfeld, New realization of Yangians and quantum affine algebras, Sov. Math. Dokl. 36 (1988), 212-216.

[6] B. Enriquez, On correlation functions of Drinfeld currents and shuffle algebras, Transformation Groups 5 (2000), no. 2, 111-120.

[7] B. Enriquez, S. Khoroshkin, S. Pakuliak, Weight functions and Drinfeld currents, Commun. Math. Phys. 276 (2007), 691-725.

[8] B. Enriquez, V. Rubtsov, Quasi-Hopf algebras associated with $\mathfrak{s l}_{2}$ and complex curves, Israel J. Math 112 (1999), 61-108.

[9] S. Khoroshkin, S. Pakuliak, Weight function for $U_{q}\left(\widehat{\mathfrak{s l}}_{3}\right)$, Theor. and Math. Phys. 145 (2005), no. 1, 1373-1399.

[10] S. Khoroshkin, S. Pakuliak, V. Tarasov, Off-shell Bethe vectors and Drinfeld currents, Journal of Geometry and Physics 57 (2007), 1713-1732.

[11] S. Khoroshkin, V. Tolstoy, On Drinfeld realization of quantum affine algebras, Journal of Geometry and Physics 11 (1993), 101-108.

[12] S. Khoroshkin, S. Pakuliak, A computation of an universal weight function for the quantum affine algebra $U_{q}\left(\widehat{\mathfrak{g l}}_{N}\right)$, J. Math. Kyoto Univ. 48, no. 2, 277-322. Preprint arXiv:0711.2819 [math.QA].

[13] S. Khoroshkin, S. Pakuliak, Generating series for nested Bethe vectors, SIGMA 4 (2008), 081, 23 pages, arXiv:0810.3131.

[14] P. Kulish, N. Reshetikhin, Diagonalization of $G L(N)$ invariant transfer matrices and quantum $N$-wave system (Lee model), J. Phys. A: Math. Gen. 16 (1983), L591-L596.

[15] E. Mukhin, V. Tarasov, A. Varchenko, Bethe eigenvectors of higher transfer matrices, J. Stat. Mech. Theory Exp. 2006, no. 8, P08002, 1-44.

[16] A. Oskin, S. Pakuliak, A. Silantyev, On the universal weight function for the quantum affine algebra $U_{q}\left(\widehat{\mathfrak{g l}}_{N}\right)$, Preprint arXiv:0711.2821 [math.QA].

[17] N. Reshetikhin, M. Semenov-Tian-Shansky, Central extentions of quantum current groups, Lett. Math. Phys. 19 (1990), 133-142.

[18] V. Tarasov, A. Varchenko, Jackson integrals for the solutions to Knizhnik-Zamolodchikov equation, Algebra and Analysis 2 (1995) no.2, 275-313.

[19] V. Tarasov, A. Varchenko, Geometry of q-hypergeometric functions, quantum affine algebras and elliptic quantum groups, Astérisque 246 (1997), 1-135.

[20] V. Tarasov, A. Varchenko, Combinatorial formulae for nested Bethe vectors, arXiv:math/0702277 [math.QA]. 
Luc Frappat and Éric Ragoucy

Laboratoire d'Annecy-le-Vieux de Physique Théorique LAPTH

UMR 5108 du CNRS associée à l'Université de Savoie

BP 110

F-74941 Annecy-le-Vieux Cedex

France

e-mail: frappat@lapp.in2p3.fr

ragoucy@lapp.in2p3.fr

Sergey Khoroshkin

Institute of Theoretical \& Experimental Physics

117259 Moscow

Russia

e-mail: khor@itep.ru

Stanislav Pakuliak

Laboratory of Theoretical Physics

JINR

141980 Dubna, Moscow reg.

Russia

e-mail: pakuliak@theor.jinr.ru

Communicated by Petr Kulish.

Submitted: October 30, 2008.

Accepted: February 16, 2009. 